\title{
Die speciellen Laméschen Functionen erster Art von beliebiger Ordnung.
}

(Von Herrn E. Heine in Halle.)

In einer Arbeit des $60^{\text {sten }}$ Bandes dieses Journals wurden Functionen eingeführt, welche ich Lamésche Functionen höherer Ordnung nannte, weil sie sich für den speciellen Werth 2 eines Parameters in diejenigen verwandeln, welche Lamé in seinen Untersuchungen über den Wärmezustand eines Ellipsoides anwandte. Es blieb noch übrig, einige Fragen năher zu erörtern, welche in jenem Aufsatze nicht mit der erforderlichen Ausführlichkeit behandelt werden konnten. Die gegenwärtige Arbeit, welche durch die neulich erschienene "Ueber einige bestimmte Integrale" vorbereitet wird, behandelt den Gegenstand, welcher Bd. 60, S. 254 und 258 berührt wurde, nämlich die speciellen Functionen der verschiedenen Ordnungen.

Bezeichnet $p$ eine ganze positive, $n$ eine ganze nicht negative Zahl, so wurde die Lamésche Funvtion $p^{\text {ter }}$ Ordnang, welche $z u n$ gehört, mit Hülfe der Differentialgleichung

$$
\frac{d^{2} W}{d u^{2}}-\left[c_{0} x^{p-1}+c_{1} x^{p-2}+\text { etc. }+c_{p-1}\right] W=0
$$

definirt, in welcher

$$
d u=\frac{d x}{\sqrt{\psi(x)}}, \quad \psi(x)=(x-\alpha)\left(x-\alpha_{1}\right) \ldots\left(x-\alpha_{p}\right)
$$

gesetzt ist. Die $\alpha$ bedeuten hier Constante, welche man sich als gegeben zu denken hat, während die c Constante sind, die so bestimmt werden müssen, dass ein Integral der Differentialgleichung eine ganze Function $n^{\text {ten }}$ Grades von $\sqrt{x-\alpha}, \sqrt{x-\alpha_{1}}$, etc., $\sqrt{x-\alpha_{p}}$ wird. Dies Integral ist dann eben die Lamésche Function $p^{\text {ter }}$ Ordnung, welche zu $n$ gehört, und zwar erster Art, indem die Function zweiter Art sich auf ein zweites Integral derselben Differentialgleichung bezieht. In der gegenwärtigen Arbeit wollen wir nur die Functionen erster Art betrachten.

Die Differentialgleichung und ihre Integrale vereinfachen sich, wie Bd. 60, S. 254 gesagt wurde, wenn mehrere Constante $\alpha$ in $\psi(x)$ gleich werden. Der Fall ist von besonderer Wichtigkeit, in welchem $\alpha_{1}=\alpha_{2}=$ etc. $=\alpha_{p}$ 
wird, und in diesem dürfen wir offenbar, ohne ihn irgend wesentlich zu beschränken,

$$
\alpha=0 ; \quad \alpha_{1}=\alpha_{2}=\text { etc. }=\alpha_{p}=1
$$

setzen. Die Functionen, welche dann durch die Differentialgleichung gegeben werden, sind die speciellen Functionen, mit denen wir uns wegen ihrer Einfachheit und der vorläufig überwiegenden Wichtigkeit hier beschäftigen wollen.

Ausser diesem extremen Falle könnte man auch den betrachten, in welchem nur einige a gleich werden; hier wird derselbe zwar ausgeschlossen, aber in einer folgenden Abhandlung näher erörtert, in welcher wir die wirkliche Existenz der allgemeinen Laméschen Functionen höherer Ordnung nachweisen. Es wird dabei erforderlich sein, über eine etwas allgemeinere Differentialgleichung zu handeln.

In dem ersten Abschnitte des vorliegenden Aufsatzes wird die Aufgabe gelöst, wegen welcher die Arbeit ursprünglich unternommen war; es werden nämlich die Constanten $c$ und damit die Differentialgleichungen der speciellen Functionen gefunden (\$.3), und endlich (\$.5) diejenigen Integrale derselben, welche ganze Functionen $n^{\text {ten }}$ Grades von $\sqrt{x-\alpha}, \sqrt{x-\alpha_{1}}$, etc., also hier von $\sqrt{ } x$ und $\sqrt{x-1}$ sind, d. h. sämmtliche specielle Functionen $p^{\text {ter }}$ 0rdnung. Es stellt sich heraus, dass für jedes $p$ genau $n+1$ solcher Functionen existiren, die $z u n$ gehören; nämlich, abgesehen von dem Falle $p=1$, der eine Modification erfordert, hat man für jedes $n$ genau $n+1$ Differentialgleichungen, von denen jede ein brauchbares Integral liefert.

Es ist bequemer, diese Functionen als abhängig nicht mehr von $x$ sondern von $z=\sqrt{ } x$ zu betrachten. Nennt man dann die $n+1$ speciellen Laméschen Functionen $p^{\text {ter }}$ Ordnung, welche zu $n$ gehören, $I_{\nu}^{n}[p, z]$, wo $\nu$ die Werthe $0,1,2$, etc. $n$ annimmt, so zeigt sich $(\$ .6)$, dass $I_{\nu}^{n}[p, z]$ sich nur durch einen constanten Factor von $\left(\sqrt{z^{2}-1}\right)^{\nu} \frac{d^{\nu} I_{0}^{n}[p, z]}{d z^{\nu}}$ unterscheidet. Es wird aber $I_{0}^{n}[p, z]$, wiederum bis auf einen constanten Factor, je nachdem $p$ grade oder ungrade, von der Form $p=2 \widetilde{\omega}+2$ oder $p=2 \widetilde{\omega}+1$ ist, nichts Anderes als der $\widetilde{\omega}^{\text {te }}$ Differentialquotient nach $z$ respective von $P^{n+\varpi}(z)$ oder $\cos ((n+\widetilde{\omega}) \arccos z)$. Für $p=2$ stimmt also $I_{\nu}^{n}[p, z]$ mit $P_{\nu}^{n}(z)$ überein.

Mitgetheilt wurde bereits Bd. 60, S. 258 der ungefähre Zusammenhang, in dem die so erhaltenen $I_{\nu}^{n}$, welche nach ihrer Entstehung eine bestimmte Rolle unter den linearen Differentialgleichungen zweiter Ordnung spielen, mit einer partiellen Differentialgleichung von $p+1$ unabhängigen 
Variabeln stehen; die Ausführung dessen, was dort angedeutet wurde, ist Gegenstand des zweiten und dritten Abschnittes.

Man weiss (m. vergl. die Einleitung des Handbuches der Kugelfunctionen), dass Laplace die reciproke Entfernung zweier Punkte mit den rechtwinkligen Coordinaten $\xi, \xi_{1}, \xi_{2}$ und $a, a_{1}, a_{2}$ - des bequemeren Ausdruckes halber setzen wir $a^{2}+a_{1}^{2}+a_{2}^{2}=1$ - mit der Differentialgleichung

$$
\frac{\partial^{2} \boldsymbol{T}}{\partial \xi^{2}}+\frac{\partial^{2} T}{\partial \xi_{1}^{2}}+\frac{\partial^{2} T}{\partial \xi_{2}^{2}}=0
$$

in Verbindung brachte. Setzt man $\dot{\xi}^{2}+\xi_{1}^{2}+\dot{\xi}_{2}^{2}=r^{2}$, und $\dot{\xi}=r x, \xi_{1}=r x_{1}, \xi_{2}=r x_{2}$, führt auch für die $x$ Polarcoordinaten $\psi_{1}$ und $\psi_{2}$ ein, entwickelt dann $T$ nach Potenzen von $r$, so wird der Coefficient von $r^{n}$, der $P^{n}$ heisst, von einem Argumente $a x+a_{1} x_{1}+a_{2} x_{2}$ abhängen, und einer neuen partiellen Differentialgleichung mit den beiden unabhängigen Veränderlichen $\psi_{1}$ und $\psi_{2}$, welche die zweite Differentialgleichung heissen mag, genügen. Laplace handelt nun von den $\boldsymbol{P}$ in doppelter Beziehung; erstens entwickelt er Eigenschaften derselben, die ganz unabhängig von ihrem Verhältnisse zur Differentialgleichung sind, und zweitens betrachtet er sie in ihrer Beziehung zu den anderen Lösungen der zweiten Differentialgleichung. Zu den Entwickelungen der ersten Art gehört z. B. die merkwürdige Reihe für $\boldsymbol{P}^{n}$, die nach Cosinus und Sinus der Vielfachen von $\psi_{2}$ fortschreitet. Unwesentlich ist es, dass Laplace auch die Eigenschaften der ersten Art mit Zugrundelegung solcher der zweiten Art ableitet; Jacobis Methode zeigt, dass die Ableitung' ebenso unabhängig von der zweiten Differentialgleichung gemacht werden kann, wie die Eigenschaften, von denen wir reden, es sind.

Die erwähnten Untersuchungen von Laplace lassen sich von drei auf $p+1$ Veränderliche übertragen. Schon im $13^{\text {ten }}$ Bande des Liouvilleschen Journals hat Cayley*) bemerkt, dass zum Zwecke einer Verallgemeinerung der Laplaceschen Functionen eine $\frac{1}{2}(p-1)^{\text {te }}$ Potenz mit der ersten partiellen Differentialgleichung, nachdem sie auf $p+1$ Variable bezogen worden ist, in Verbindung zu bringen sei, nämlich der Ausdruck $T$ im $\$ \$ \$ 19$ mit der dort befindlichen Gleichung (5.). Im Folgenden führen wir die Uebertragung aus; die Verbindung mit dem ersten Abschnitte wird, wie schon gesagt, dadurch hergestellt, dass hierbei die Functionen $I_{\nu}^{n}$ wieder auftreten.

In dem zweiten Abschnitte findet man die Resultate, welche sich für die Functionen, welche aus der Entwickelung der $\frac{1}{2}(p-1)^{\text {ten }}$ Potenz entspringen,

*) Sur les fonctions de Laplace, p. 275-280. 
unabhängig von ihrer Beziehung zur Differentialgleichung ergeben. Wenn ich hierbei die Jacobische Methode zu Grunde lege, so geschieht dies nicht nur um eine bessere Anordnung zu erzielen; man wird die Vorzüge der Jacobischen Methode erkennen, wenn man nach Anleitung des \$. 21 dieselben Resultate mit Hülfe der Differentialgleichung abzuleiten versucht, indem man das Verfahren von Laplace nachbildet. Ich möchte sagen, dass man die ungefähre Form der Resultate leichter bei dem letzten Verfahren erräth, die genauen Resultate und strenge Beweise aber, sobald $p>2$ ist, aus dem Jacobischen erhält. Indem wir später, um den Inhalt des dritten Abschnittes genauer auseinanderzusetzen, an dieser Stelle wieder anknüpfen, kommen wir nun zu der speciellen Angabe des Inhaltes im zweiten Abschnitte.

Wir gehen von der Entwickelung der Grösse

$$
\frac{1}{\left(1-2 r z+r^{2}\right)^{\frac{1}{2}(p-1)}}
$$

nach aufsteigenden Potenzen von $r$ aus. Heisst der Coefficient von $r^{n}$ bis auf eine Constante ('Taf. I) $I^{n}[p, z]$, so zeigt man leicht (\$. 9), dass dieser durch denselben $\widetilde{\omega}^{\text {ten }}$ Differentialquotienten dargestellt wird wie oben $I_{0}^{n}$, eine Darstellung von $I^{n}[p, z]$, welche, wie ich aus einem Schreiben des Herrn Mehler ersah, diesem bereits vor Abfassung meiner Arbeit über bestimmte Integrale bekannt war. Für $p=2$ ist $I^{n}[p, z]$ offenbar nichts Anderes als $P^{n}(z)$. Man erkennt, dass die $I^{n}[p, z]$ und daher die $I_{\nu}^{n}[p, z]$ wesentlich nur zwei verschiedene Charaktere haben; für grade $p$ führen sie auf Kugelfunctionen, für ungrade $p$ auf Kreisfunctionen. Ein Blick auf Taf. I-III lehrt diese Beziehungen. Die $I_{\nu}^{n}$ spielen in Bezug auf $I^{n}$ dieselbe Rolle wie die Zugeordneten $\boldsymbol{P}_{\nu}^{n}$ in Bezug auf $\boldsymbol{P}^{n}$.

Es wird nun gezeigt, wie die wichtigsten Eigenschaften der Kugelund Kreisfunctionen sich bei den $I$ wiederfinden. Wir erwähnen nur flüchtig die zweite Formẹl in Taf. V, deren Bedeutung man sofort aus der darüberstehenden von Jacobi herrührenden erkennt; Taf. VI drückt die $I^{n}$ durch Integrale aus, die den Laplaceschen für $P^{n}$ im Falle $p=2$ entsprechen, und die meiner Arbeit über bestimmte Integrale Bd. 61 entnommen sind; auch die Bedeutung der Formeln in Taf. VII ist selbstverständlich, wenn man bedenkt, dass $I_{\nu}^{n}$ für $p=2$ in $P_{v}^{n}$ übergeht; erst die Formeln der Taf. VIII geben zu einer ausführlicheren Erörterung Anlass.

Sieht man bei dieser Uebersicht immer von constanten Factoren ab, so ist, wie ich nachwies (Handb. $\$ .43$, S.117), der Coefficient von $\cos m \psi$ in der 
Entwickelung von

$$
\left(x+\cos \psi \sqrt{x^{2}-1}\right)^{n}
$$

nach Cosinus der Vielfachen von $\psi$ gleich $P_{m}^{n}(x)$; dies in die allgemeineren Zeichen $I$ übersetzt, heisst, wenn man $\cos \psi=z$ macht: Der Coefficient von $I^{m}[1, z]$ in der Entwickelung unseres Ausdruckes nach $I^{m}[1, z]$ mit veränderlichem $m$ ist die Zugeordnete nächst höherer Ordnung $I_{m}^{n}[2, x]$. Nach den

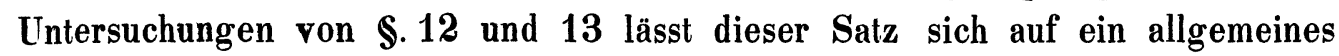
$p$ so übertragen, dass man für jene $x^{\text {te }}$ Potenz die Entwickelung erhält:

$$
\left(x+z \sqrt{x^{2}-1}\right)^{n}=\sum_{m=10}^{m=n} b_{1 n} I_{m}^{n}[p+1, x] I^{m}[p, z],
$$

wenn die $b$ gewisse Constante bezeichnen, deren Werth man in Taf. VII findet.

Während man für $p=1$ wieder auf die frühere Formel zurückkommt, erhält man für $p=2$ etwas wesentlich Neues, nämlich die elegante Entwickelung unserer $n^{\text {ten }}$ Potenz statt nach Cosinus der Vielfachen von $\arccos z$, nach Kugelfunctionen $P^{m}(z)$; der Factor von $P^{m}(z)$ ist aber eine Zugeordnete dritter Ordnung $I_{\nu}^{n}[3, x]$. Die für höhere $p$ geltenden Formeln lassen sich übrigens auch durch blosse Differentiation nach $z$ aus den beiden fundamentalen (für $p=1$ und $p=2$ ) ableiten.

Wir kommen zu einer zweiten Reihe, nämlich zu der Entwickelung von $P^{\mu}\left(\cos \gamma_{1}\right)$ nach Cosinus der Vielfachen von $\gamma_{2}$, wenn man setzt

$$
\cos \gamma_{1}=\cos \eta_{1} \cos \psi_{1}+\sin \eta_{1} \sin \psi_{1} \cos \gamma_{2} \text {. }
$$

Bekanntlich tritt in dieser Laplaceschen Reihe als Factor von $\cos m \gamma_{2}$ das Product $\boldsymbol{P}_{m}^{n}\left(\cos \eta_{1}\right) P_{m}^{n}\left(\cos \psi_{1}\right)$ auf, oder in unseren allgemeinen Zeichen, es ist für $p=2$ in der Entwickelung von $I^{n}\left[p, \cos \gamma_{1}\right]$ nach $I^{m}\left[p-1, \cos \gamma_{2}\right]$ mit veränderlichem $m$, der Factor von $I^{m}\left[p-1, \cos \gamma_{2}\right]$ ein Product der Zugeordneten nächsthöherer Ordnung

$$
I_{m}^{n}\left[p, \cos \eta_{1}\right] I_{m}^{n}\left[p, \cos \psi_{1}\right]
$$

Aus \$.14-16 ersieht man, dass dasselbe Resultat für alle $p$ gilt, die grösser als 1 sind.

Die Fälle $p=2$ und $p=3$ sind hier die fundamentalen; im $\$ .17$ sieht man, wie wichtig es ist, diese Formel für jedes grössere $p$ zu besitzen, indem man dann eine noch allgemeinere Reihe für $I^{n}\left[p, \cos \gamma_{1}\right]$ erhält, wo $\gamma_{1}$ durch $p-1$ Winkel $\eta$, eben so viele $\psi$ und einen Winkel $\gamma_{p}$ ausgedrückt ist. Wollte man für $\gamma_{1}$ nicht wie dort $2 p-1$ sondern eine grössere Anzahl von Winkeln einführen, indem man fortfährt und $\gamma_{p}$ wiederum durch Winkel $\eta_{p}$, 
$\psi_{p}$ und $\gamma_{p+1}$ ausdrückt, so würde man nicht mehr ein einfaches Resultat erhalten. Man sieht hieraus, dass man für $p=2$ nur drei Winkel einführen durfte, wie es auch Laplace that (oder vier, indem man für $\gamma_{2}$ die Differenz $\psi_{2}-\eta_{2}$ setzt).

Dies ist der hauptsächliche Inhalt des zweiten Abschnittes; wir kommen jetzt zum dritten Abschnitte. Indem man statt der reciproken Quadratwurzel den Ausdruck $T$ im $\$$. 19 betrachtet, welcher nun einer ersten Gleichung (5.) mit $p+1$ statt drei Veränderlichen genügt, findet man eine zweite Gleichung (6.), welcher die Function

$$
I^{n}\left[p, a x+a_{1} x_{1}+\text { etc. }+a_{p} x_{p}\right]
$$

genügt. Die Bedeutung der verschiedenen Buchstaben, welche genau der oben für $p=2$ gewählten entspricht, ist im $\$ .19$ vollständig angegeben. Wir suchen nun die allgemeinste Function $X^{(n)}$, welche mit $I^{n}$ folgende Eigenschaften gemein hat: Sie ist vom Grade $n$ in Bezug auf $x, x_{1}$, etc. $x_{p}$, und genügt der Gleichung (6.).

Um die Function aufzufinden, führt man Polarcoordinaten $\psi_{1}, \psi_{2}$, etc. ein (\$.19), und stellt eine endliche Function $Z$ auf (\$.20), welche von den $\psi$ abhängt, und aus einer Summe von Gliedern besteht, deren jedes einzelne (6.) genügt, die übrigens nicht die allgemeinste Function ist, welche diese Eigenschaften besitzt. Im $\mathbb{S} .22$ zeigt sich, dass dieses $Z$ eine ganze Function $n^{\text {ten }}$ Grades der $x$ ist, im $\mathfrak{S} .23$ endlich, dass sie so viele Constanten enthält als $X^{(n)}$ höchstens enthalten kann. Es ist daher $Z$ jene allgemeinste Function $X^{(n)}$.

Endlich findet man im $\$ .22$ noch die Sätze, welche eine Haupteigenschaft der $X^{(n)}$ enthalten; nachdem gezeigt ist, dass $Z$ mit $X^{(n)}$ übereinstimmt, kann man nämlich dort in $(a)$ und $(b)$ die Buchstaben $Z$ und $Y$ mit $X^{(n)}$ und $\boldsymbol{X}^{(m)}$ vertauschen. Die Bedeutung der beiden Sätze wird aus der Vergleichung des Resultats mit dem für $p=2$ geltenden sofort klar; sie sind es., durch welche die Coefficientenbestimmung geschieht, wenn man eine Function in eine Reihe entwickeln will, die nach den $X^{(n)}$ fortschreitet.

Die Versuche zur Verallgemeinerung der Laplaceschen Functionen, soweit sie mir bekannt geworden sind, haben sich bisher auf diese beiden Sätze bezogen, die man in Cayleys oben erwähnter Arbeit S. 276 und 278 Formel 10 und 16 bereits findet. Besonders bemerkenswerth scheint mir in dieser Arbeit die Art zu sein, auf welche Cayley gelehrt hat, die $X^{(n)}$ ohne Hülfe unserer Gleichung (6.), nur mit Hülfe von (5.) zu definiren. Wenn wir diesen Vorgang nicht benutzten, so geschah es aus dem Grunde, weil 
uns daran lag, auch die Reihenentwickelung von $X^{(n)}$ (m. vergl. \$. 23) und die Art kennen zu lernen, auf welche die Constanten in $X^{(n)}$ eingehen.

Im Falle $p=2$ hatten wir eine erste (5.) entsprechende Differentialgleichung und eine zweite unterschieden, welche (6.) entspricht. Jedes Integral der zweiten, mit $\boldsymbol{r}^{n}$ multiplicirt, muss offenbar ein Integral der ersten sein. Die Laplacesche Function $X^{(n)}$ (für $p=2$ ) lässt sich, wie man bemerkt hatte, dadurch definiren, dass sie ganz und vom Grade $n$ nach $x, x_{1}, x_{2}$ ist und der zweiten Differentialgleichung genügt. Nimmt man den neuen Gedanken hinzu, der wohl Cayley angehört, dass man sie auch als homogene Function der $x$ betrachten kann, so sieht man, dass $r^{n^{\prime}} X^{(n)}$ die allgemeinste Function ist, welche der ersten Gleichung genügt, und homogen und vom Grade $n$ nach $\xi, \xi_{1}, \xi_{2}$, dass also $X^{(n)}$ dieselbe Function für $\xi^{2}+\xi_{1}^{2}+\xi_{2}^{2}=1$ wird. Cayley verallgemeinert nun den Begriff der Laplaceschen Function dahin, dass sie die allgemeinste homogene Function $n^{\text {ten }}$ Grades von $\xi$, $\xi_{1}$, etc. $\xi_{p}$ ist, welche unserer Gleichung (5.) genügt, wenn man $\xi^{2}+\xi_{1}^{2}+$ etc. $+\xi_{p}^{2}=1$ setzt. Diese Function stimmt nun mit unserem $X^{(n)}$ überein; Cayley beweist für dieselbe die in Rede stehenden beiden Sätze, geht übrigens weder näher auf die definitive Form von $X$ noch auf den Nachweis ein, dass die von ihm S. 276 aufgestellten Functionen wirklich die allgemeinsten, von ihm definirten $X^{(n)}$ sind.

Mit dem Beweise der erwähnten Sätze hat sich ferner auch Ostrogradski im Bulletin de l'Académie impériale des sciences de St. Petershourg Tome III, p. 65 vom Juni $1860^{\prime}$ in einer Arbeit: Sur une intégrale définie beschäftigt, indem er für die $X^{(n)}$ die Cayleysche Definition zu Grunde legte und die Methode benutzte, durch die ein Integral, welches sich über einen Körper erstreckt, in ein über die Oberfläche genommenes verwandelt wird.

\section{Erster Abschnitt.}

§. 1. Wie bereits in der Einleitung angegeben ist, haben wir die Differentialgleichung

$$
\text { (1.) } \frac{d^{2} W}{d u^{2}}-\left[c_{0} x^{p-1}+c_{1} x^{p-2}+\text { etc. }+c_{p-1}\right] W=0
$$

zu untersuchen, in welcher man

$$
d u=\frac{d x}{\sqrt{\psi(x)}}, \quad \psi(x)=(x-\alpha)\left(x-\alpha_{1}\right) \ldots\left(x-\alpha_{p}\right)
$$


gemacht hat. Jede Function $W$, welche ganz und vom Grade $n$ in Bezug auf $\sqrt{x-\alpha}, \sqrt{x-\alpha_{1}}$, etc., $\sqrt{x-\alpha_{p}}$ ist, lässt sich jedenfalls als eine Summe

$$
W=G_{1} \sqrt{ } \psi_{1}+G_{2} \sqrt{ } \psi_{2}+\text { etc. }
$$

darstellen, in der die $\boldsymbol{G}$ ganze Functionen von $x$, die Functionen $\psi_{1}, \psi_{2}$, etc. Theiler von $\psi$ sind, die extremen Fälle eingeschlossen, dass eine der $\psi_{1}$, etc. gleich 1 oder gleich $\psi(x)$ selbst ist. Offenhar kann man annehmen, dass $\psi_{1}, \psi_{2}$, etc. untereinander verschieden sind, d. h. dass der Quotient von zwei derselben keine Constante giebt. Dies vorausgesetzt zeigen wir, dass jede einzelne Grösse $\left.G_{1}\right\urcorner \psi_{1}, G_{2} \downarrow \psi_{2}$, etc., für $W$ in (1.) eingesetzt, dieser Gleichung genügen muss.

Da nämlich

$$
\frac{d^{2} W}{d u^{2}}=\psi(x) \frac{d^{2} W}{d x^{2}}+\frac{1}{2} \psi^{\prime}(x) \frac{d W}{d x},
$$

und daher für jeden Index ı

$$
\frac{d^{2}\left(G_{\iota} \sqrt{\psi_{\iota}}\right)}{d u^{2}}=\boldsymbol{H}_{\iota} \sqrt{ } \psi_{\iota}
$$

wird, wo $H_{\iota}$ eine rationale Function von $x$ bezeichnet, so erfordert (1.), dass

$$
\boldsymbol{H}_{1} / \psi_{1}+\boldsymbol{H}_{2} / \psi_{2}+\cdots
$$

gleich einem Ausdrucke

$$
\boldsymbol{K}_{1} \sqrt{ } \psi_{1}+\boldsymbol{K}_{2} \sqrt{ } \psi_{2}+\cdots
$$

werde, wo die $K$ ganze Functionen von $x$ vorstellen, nämlich solche, dass man hat

$$
K_{\iota}=G_{\iota}\left[c_{0} x^{p-1}+c_{1} x^{p-2}+\text { etc. }+c_{p-1}\right] .
$$

Hieraus schliesst man sofort, dass

$$
\boldsymbol{H}_{\iota}=\boldsymbol{K}_{\iota}, \quad \boldsymbol{H}_{\iota} \sqrt{ } \psi_{\iota}=\boldsymbol{K}_{\iota} / \psi_{\iota}
$$

wird, dass also $G_{\iota} \downarrow \psi_{\iota}$ der Gleichung (1.) genügt. Wir haben also das Resultat: Soll man alle Functionen $W$ aufsuchen, welche (1.) genügen, und ganze Functionen $n^{\text {ten }}$ Grades in Bezug auf $\sqrt{x-\alpha}, \sqrt{x-\alpha_{1}}$, etc., $\sqrt{x-\alpha_{p}}$ sind, so genügt es, alle Functionen $W$ von der Form $W=G \sqrt{ } \psi_{1}$ aufzusuchen, die (1.) genügen. Hier bezeichnet $\psi_{1}$ irgend einen Theiler von $\psi, G$ eine ganze Function von $x$ von solchem Grade, dass $W^{2}$ eine (selbstverständlich ganze) Function von $x$ vom Grade $n$ wird.

Hat man alle diese Functionen gefunden, so ist offenbar der allgemeinste Ausdruck von $W$ eine lineare Verbindung der gefundenen Functionen. 
\$. 2. Nachdem wir unsere Aufgabe so reducirt haben, dass wir also nur Integrale der Form $G \sqrt{ } \psi_{1}$ von (1.) zu betrachten brauchen, gehen wir zur Bestimmung der Constanten $c$ in der Gleichung (1.) über. In diesem Paragraphen beschäftigen wir uns mit der Bestimmung von $c_{11}$.

Setzt man in (1.) für $W$ seinen Werth $G \sqrt{ } \psi_{1}$, und macht $\psi^{\prime}=\psi_{1} \psi_{2}$, so entsteht für $G$ die Gleichung

$$
\text { (2.) } \quad \psi(x) \frac{d^{2} G}{d x^{2}}+\chi(x) \frac{d G}{d x}+\vartheta(x) G=0,
$$

wenn man zur Abkürzung

$$
\begin{aligned}
& \chi(x)=\frac{1}{2} \psi^{\prime}(x)+\psi_{1}^{\prime}(x) \psi_{2}(x), \\
& \boldsymbol{\vartheta}(\boldsymbol{x})=\frac{1}{2} \psi_{1}^{\prime \prime}(\boldsymbol{x}) \psi_{2}(\boldsymbol{x})+\frac{1}{4} \psi_{1}^{\prime}(\boldsymbol{x}) \psi_{2}^{\prime}(\boldsymbol{x})-\left[\boldsymbol{c}_{0} \boldsymbol{x}^{p-1}+\text { etc. }+\boldsymbol{c}_{p-1}\right]
\end{aligned}
$$

gemacht hat. Es ist nun $\psi(x)$ vom Grade $p+1$; es sei $\psi_{1}$ vom Grade $\nu$, also $\psi_{2}$ vom Grade $p+1-\nu$. Wegen des Grades von $W$ ist dann der Grad der ganzen Function $G$ von $x$ gleich $\frac{1}{2}(n-\nu)$, welches wir gleich $\sigma$ setzen. Integrirt man nun (2.) durch eine nach Potenzen von $x$ absteigende Reihe, die also mit (der ganzen Potenz) $x^{\sigma}$ anfängt, so muss die höchste Potenz von $x$, welche auf der linken Seite von (2.) nach Einsetzung der Reihe für $G$ entsteht, sich von selbst fortheben. Diese, die $p+\sigma-1^{\text {te }}$, kommt in

$$
\begin{array}{ccc}
\psi(x) \frac{d^{2} G}{d x^{2}}, & \chi(x) \frac{d G}{d x}, & \vartheta(x) G ; \\
\sigma(\sigma-1), & \sigma\left(\nu+\frac{p+1}{2}\right), & \frac{\nu(\nu+p-1)}{4}-c_{0}
\end{array}
$$

resp.

mal, also im ganzen $\frac{n(n+p-1)}{4}-c_{0}$ mal vor, d. h. es ist nothwendig

$$
c_{0}=\frac{n(n+p-1)}{4} \text {. }
$$

\$. 3. Die übrigen Constanten c ermitteln wir nur für die speciellen Functionen. Wir setzen also

$$
\alpha=0 ; \quad \alpha_{1}=\alpha_{2}=\text { etc. }=\alpha_{p}=1,
$$

so dass $\psi(x)$ gleich $x(x-1)^{p}$ wird, und $W$, nach $\$$. 1 , jedenfalls von einer der vier Formen

Ferner ist nun

$$
G ; \quad G \sqrt{ } x ; \quad G \sqrt{x-1} ; \quad G \sqrt{ } x \sqrt{x-1}
$$

$$
\frac{d^{2} W}{d u^{2}}=x(x-1)^{p} \frac{d^{2} W}{d x^{2}}+\frac{(p+1) x-1}{2}(x-1)^{p-1} \frac{d W}{d x} \text {. }
$$

Man hat jetzt zwei Fälle zu unterscheiden; erstens kann es eintreten, dass $W$ für $x=1$ nicht verschwindet, und dann muss der Factor von $W$ in 
(1.), nämlich

$$
c_{0} x^{p-1}+c_{1} x^{p-2}+\cdots+c_{p-1}
$$

ganz theilbar sein durch $(x-1)^{p-1}$, also gleich $c_{0}(x-1)^{p-1}$. Dividirt man nun alle Glieder von (1.) durch den gemeinsamen Factor, so findet man, mit Benutzung des schon aus $\$$. 2 bekannten Werthes von $c_{0}$, folgende Differentialgleichung für die speciellen Functionen erster Art und $p^{\text {ter }}$ Ordnung, die zu $n$ gehören, wenn sie für $x=1$ nicht verschwinden:

$$
x(x-1) \frac{d^{2} W}{d x^{2}}+\frac{(p+1) x-1}{2} \frac{d W}{d x}-\frac{n(n+p-1)}{4} W=0 .
$$

Verschwindet zweitens $W$ für $x=1$, so ist es durch eine Potenz von $\sqrt{x-1}$ theilhar; es sei diese die $\nu^{\text {te }}$, wo $\nu$ also eine ganze positive, $n$ nicht überschreitende Zahl vorstellt. $W$ soll ausserdem durch keine höhere Potenz von $\sqrt{x-1}$ als die $\nu^{\text {te }}$ theilbar sein. Dann sind

$$
(x-1)^{p} \frac{d^{2} W}{d x^{2}} ; \quad(x-1)^{p-1} \frac{d W}{d x} ; \quad \frac{d^{2} W}{d u^{2}}
$$

durch die $\frac{1}{2} \nu+p-2^{\text {ie }}$ Potenz von $x-1$ theilbar, woraus unmittelbar folgt, dass der Factor von $W$ in (1.) durch $(x-1)^{p-2}$ theilbar, also von der Form ist:

$$
(x-1)^{p-2}\left[\frac{n(n+p-1)}{4} x+k\right] \text {, }
$$

wenn $k$ eine Constante nach $x$ vorstellt. Die Gleichung solcher Functionen $W$ ist also

$$
4 x(x-1)^{2} \frac{d^{2} W}{d x^{2}}+2[(p+1) x-1](x-1) \frac{d W}{d x}-[n(n+p-1) x+k] W=0 .
$$

Aber auch $k$ kann nicht willkürlich gewählt werden; führt man nämlich in die vorstehende Gleichung eine für $x=1$ nicht verschwindende Grösse $U$ ein, indem man setzt

so findet man für $U$ die Gleichung

$$
W=(x-1)^{\frac{1}{2} \nu} U,
$$

$$
4 x(x-1)^{2} \frac{d^{2} U}{d x^{2}}+2[(p+2 \nu+1) x-1](x-1) \frac{d U}{d x}+[x(\nu(\nu+p-1)-n(n+p-1))-k-\nu] U=0 .
$$

Da in dieser für $x=1$ die beiden ersten Glieder verschwinden, so muss auch das letzte Glied, also in demselben der Factor von $U$, für $x=1$ verschwinden, und man erhält daher als Werth von $k$

$$
k=\nu(\nu+p-2)-n(n+p-1),
$$

welcher in die Gleichungen für $U$ und $W$ einzusetzen ist. Dividirt man diese nun noch durch $x-1$, so hat man schliesslich das Resultat: 
Alle speciellen, zu $n$ gehörenden Laméschen Functionen erster Art und $\boldsymbol{p}^{\text {ter }}$ Ordnung findet man, indem man untersucht, wie viele Differentialgleichungen (3.) $4 x(x-1) \frac{d^{2} W}{d x^{2}}+2[(p+1) x-1] \frac{d W}{d x}-\left[n(n+p-1)+\frac{v(v+p-2)}{x-1}\right] W=0$

ein oder mehrere Integrale $W=U(\sqrt{x-1})^{\nu}$ haben, wo $U$ eine ganze Function von $\gamma \boldsymbol{x}$ ist (deren Grad nach $\gamma \boldsymbol{x}$ dann von selbst der erforderliche $n-v$ wird). Solche Untersuchungen hat man für $v=0,1,2$, etc., $n$ zu führen; alle Functionen $W$, die man so findet, und nur diese, sind die verlangten speciellen Functionen.

Unten zeigen wir, dass für jedes $\nu$ ein Integral von (3.) mit der geforderten Eigenschaft existirt, dass man also $n+1$ specielle Functionen erster Art und $p^{\text {ter }}$ Ordnung für jedes $n$ findet. In dem Falle $p=1$ erhält man zwar nur $n$ Differentialgleichungen (3.), da für $\nu=0$ und $\nu=1$ dieselbe Gleichung (3.) hervorgeht; diese hat aber sowohl ein Integral von der Form $U$ als von der Form $U \sqrt{x-1}$, so dass man wieder $n+1$ Functionen findet.

\$. 4. Es ist bequemer unsere Functionen statt von $x$, von $z=\sqrt{ } x$ abhängig zu machen. Wir transformiren deshalb unsere Gleichungen in $z$, fügen auch zur Vervollständigung noch die Formen hinzu, die man durch Einführung einer trigonometrischen Grösse, von $\cos \psi$ statt $z$, erhält. Wir haben dann folgendes Schema:

$$
\begin{gathered}
\checkmark x=\dot{z} ; \quad z=\cos \psi ; \quad W=\left(z^{2}-1\right)^{\frac{2}{2}} U ; \\
\frac{d}{d z} \cdot\left[\left(z^{2}-1\right)^{\frac{2}{2} p} \frac{d W}{d z}\right]-\left(z^{2}-1\right)^{\frac{1}{2}(p-2)}\left[n(n+p-1)+\frac{\nu(\nu+p-2)}{z^{2}-1}\right] W=0 \\
\left(\frac{1}{\sin \psi}\right)^{p-1} \frac{d}{d \psi} \cdot\left[\sin ^{p-1} \psi \frac{d W}{d \psi}\right]+\left[n(n+p-1)-\frac{\nu(\nu+p-2)}{\sin ^{2} \psi}\right] W=0 \\
(4 .) \quad\left(z^{2}-1\right) \frac{d^{2} U}{d z^{2}}+(p+2 \nu) z \frac{d U}{d z}-(n-\nu)(n+\nu+p-1) U=0 .
\end{gathered}
$$

Es ist also noch zu zeigen, dass, bei festgehaltenem $n$ und $p$, für jeden der $n+1$ Werthe von $\nu$ eine und nur eine ganze Function $U$ der Gleichung (4.) genügt; der Fall $p=1$ macht hier keine Ausnahme.

\$. 5. Integrirt man die Gleichung (4.) durch Reihen, welche nach absteigenden Potenzen von z geordnet sind, so sieht man sogleich ein, dass die eine von ihnen mit der $n-\nu^{\text {ten }}$ Potenz, die andere mit der $-(n+\nu+p-1)^{\text {ten }}$ Potenz von $z$ beginnt. Die letztere, da sie keinenfalls eine ganze Function von $z$ ist, kann kein Integral $U$ mit den verlangten Eigenschaften geben; sie 
wäre zu benutzen, wenn wir von den Functionen zweiter Art, welche also den Kugelfunctionen $Q$ und nicht $P$ entsprechen, handeln wollten. Die erste Reihe dagegen bricht $a b$, ist daher eine ganze Function von $z$, und zwar $\Im_{v}^{n}[p, z]$, wenn man setzt

$\Im_{\nu}^{n}[p, z]=z^{n-\nu}-\frac{(n-v)(n-v-1)}{2(2 n+p-3)} z^{n-\nu-2}+\frac{(n-v)(n-\nu-1)(n-v-2)(n-\nu-3)}{2.4(2 n+p-3)(2 n+p-5)} z^{n-\nu-4}$-etc.

Macht man noch

$$
I_{\nu}^{n}[p, z]=\left(\sqrt{z^{2}-1}\right)^{\nu} \Im_{\nu}^{n}[p, z],
$$

so hat man folgendes Resultat der Untersuchungen dieses Abschnittes:

Es sind die $n+1$ Ausdrücke

$$
\text { - } I_{0}^{n}[p, z] ; \quad I_{1}^{n}[p, z] ; \quad \text { etc.; } \quad I_{n}^{n}[p, z]
$$

die sämmtlichen speciellen Laméschen Functionen erster Art und $p^{\text {ter }}$ Ordnung, welche zu n gehören.

§. 6. Die Grössen $I$ lassen sich noch durch verschiedene andere Formeln darstellen; wir heben hier die folgende hervor:

$$
I_{\nu}^{n}[p, z]=\frac{\left(z^{2}-1\right)^{\frac{1}{2}}}{n(n-1) \ldots(n-\nu+1)} \frac{d^{\nu}}{d z^{\nu}} \cdot\left[I_{0}^{n}[p, z]\right],
$$

welche zeigt, dass $I_{v}^{n}$ für alle Ordnungen $p$ durch dieselben Operationen aus $I_{0}^{n}$ abgeleitet wird, also dass $I_{\nu}^{n}$ in Bezug auf $I_{0}^{n}$ immer dieselbe Rolle spielt wie für $p=2$, d. h. wie $P_{v}^{n}$ in Bezug auf $P_{0}^{n}$. Diese Formel findet man ganz direct, wenn man die Reihe für $I_{0}^{n}$ im Ganzen $\nu$ mal nach $z$ differentiirt, aber eben so leicht aus der Bemerkung, dass die Gleichung (4.), nach $z$ differentiirt, eine Gleichung derselben Art giebt, die aus (4.) entsteht, wenn man in derselben $U$ und $\nu$ mit $\frac{d U}{d z}$ und $\nu+1$ vertauscht.

Der Character von $I_{\nu}^{n}$ hängt also immer von demjenigen von $I_{0}^{n}$ ab; dieser ist aber ein wesentlich verschiedener, je nachdem die Ordnung $p$, wie bei den Kugelfunctionen, grade oder ungrade ist. Für ein grades $p$, also für $p=2 \widetilde{\omega}+2$, lässt sich $I_{0}^{n}[p, z]$ offenbar durch $\widetilde{w}$ fache Differentiation nach $z$ aus $I_{0}^{n+\varpi}[2, z]$, d. h. aus der Kugelfunction $P^{n+\varpi}(z)$ ableiten; ist dagegen $p=2 \widetilde{\omega}+1$, durch $\widetilde{\omega}$ fache Differentiation aus $I_{0}^{n+\varpi}[1, z]$. Nach $\$ .5$ wird aber $I_{0}^{n}[1, z]$ durch die Reihe

$$
z^{n}-\frac{n(n-1)}{2(2 n-2)} z^{n-2}+\frac{n(n-1)(n-2)(n-3)}{2.4(2 n-2)(2 n-4)} z^{n-4}-\text { etc., }
$$

d. h. durch $2^{1-n} \cos (n \cdot \arccos z)=2^{1-n} \cos n \psi$ dargestellt $(\dot{z}=\cos \psi)$, so dass für das ungrade $p$ also $I_{0}^{n}[p, z]$ durch $\widetilde{w}$ fache Differentiation nach $z$ aus Journal für Mathematik Bd. LXII. Heft 2. 
$\cos (n+\varpi) \psi$ sich erzeugen lässt. Man findet Weiteres hierüber in dem zweiten Abschnitte $\$$. 9.

\$. 7. Da sowohl $P^{n}(z)$ als $\cos n \psi$ als Differentialquotient einer ganzen Potenz von $\sqrt{z^{2}-1}$ dargestellt werden können, so sind sämmtliche $I_{\nu}^{n}$ wesentlich nichts Anderes als Differentialquotienten solcher ganzen Potenzen. Benutzt man zur Integration von (4.) die Formeln des $\$$. 37 im Handb. und setzt dort in $(a$.)

$$
a=0 ; \quad b=p+2 \nu ; \quad x=z
$$

und $n-\nu$ für $n$, so wird eine ganze Function, welche (4.) integrirt, für grade oder ungrade $p$,

$$
U=\left(z^{2}-1\right)^{-\frac{2 \nu+p-2}{2}} \frac{d^{n-\nu}}{d z^{n-\nu}} \cdot\left[\left(z^{2}-1\right)^{\frac{2 n+p-2}{2}}\right],
$$

so dass man auch erhält:

$$
I_{\nu}^{n}[p, z]=\frac{\Pi(n+\nu+p-2)}{I I(2 n+p-2)}\left(z^{2}-1\right)^{-\frac{p-2}{2}} \frac{d^{n-\nu}}{d z^{n-\nu}} \cdot\left[\left(z^{2}-1\right)^{\frac{2 n+p-2}{2}}\right] .
$$

\section{Zweiter Abschnitt.}

§. 8. Entwickelt man $\left(1-2 r z+r^{2}\right)^{\frac{1-p}{2}}$ nach aufsteigenden Potenzen von $r$, und setzt

$$
\frac{1}{\left(1-2 r z+r^{2}\right)^{\frac{p-1}{2}}}=\frac{\sqrt{ } \pi}{\Pi \frac{p-3}{2}} \sum_{n=0}^{n=\infty} r^{n} I^{n}[p, z],
$$

so wird, wie man aus dem Handb. $\$ .65$, S. 169 weiss,

$$
I^{n}[p, z]=\frac{2^{n}}{\sqrt{\pi}} \cdot \frac{\Pi \frac{2 n+p-3}{2}}{\Pi n}\left(z^{n}-\frac{n(n-1)}{2(2 n+p-3)} z^{n-2}+\text { etc. }\right) \text {. }
$$

Für die in Parenthesen eingeschlossene Reihe ist aber bereits im $\mathfrak{\$} .5$ eine Bezeichnung gewählt worden, aus der sich ergiebt:

$$
I^{n}[p, z]=\frac{2^{n}}{\sqrt{\pi}} \cdot \frac{\Pi \frac{2 n+p-3}{2}}{\Pi n} I_{0}^{n}[p, z] .
$$

Die Functionen $I_{0}^{n}$ des $\$ .5$ unterscheiden sich also nur durch eine Constante von den obigen Entwickelungscoefficienten. 
Diese Festsetzungen gelten, so lange die Zahl $p$ grösser als 1 ist; um die Formeln, welche wir gewinnen werden, auch auf den Fall $p=1$ anwenden zu können, machen wir, wie man schon aus der Analogie unserer Ausdrücke mit den bei Betrachtung des Potentials entstehenden vermuthen wird,

$$
-\log \left(1-2 r z+r^{2}\right)=\gamma / \sum_{n=1}^{n=\infty} r^{n} I^{n}[1, \pi] \text {. }
$$

Wir setzen endlich fest, dass $n I^{n}[1, z]$ für $n=0$ nichts Anderes als $\frac{1}{\sqrt{ } \pi}$ vorstellt.

\$. 9. Es brauchte im vorigen Paragraphen nicht angenommen zu werden, dass $p$ ganz ist; tritt aber dieser Fall ein, so gewinnt man $I^{n}[p, z]$ sehr leicht durch Differentiation aus seinen Werthen für $p=1$ und $p=2$. (I. vergl. \$. 6.)

Ist erstens $p$ ungrade und von der Form $p=2 \widetilde{\sigma}+1$, so differentiire man

$$
-\log \left(1-2 r z+r^{2}\right) \cdot=2 \sum_{n=0}^{n=\infty} \frac{r^{n}}{n} \cos (n \cdot \arccos z)
$$

im Ganzen $\widetilde{\sigma}$ mal nach $z$; dadurch entsteht

$$
\frac{2.4 \ldots(2 \varpi-2) r^{\varpi}}{\left(1-2 r z+r^{2}\right)^{\varpi}}=\sum \frac{r^{n}}{n} \frac{d^{\varpi} \cos (n \cdot \arccos z)}{d z^{\varpi}}
$$

also mit Hülfe des $\$$. 8

$$
(n+\widetilde{\omega}) 2^{\varpi} I^{n}[2 \widetilde{\omega}+1, z]=\frac{2}{\sqrt{ } \pi} \frac{d^{n+\varpi} \cos ((n+\varpi) \arccos z)}{d z^{\varpi}} .
$$

Wir bezeichnen nun eine parallel mit $\boldsymbol{P}$ vorkommende trigonometrische Grösse durch $C$, indem wir setzen

$$
C^{n}(z)=I^{n}[1, z]=\frac{2}{n \sqrt{ } \pi} \cos (n \arccos z),
$$

für $n=0$ rechts die Hälfte genommen. Dann wird

$$
2^{\varpi} I^{n}[2 \widetilde{\omega}+1, z]=\frac{d^{\varpi} C^{n+\varpi}(z)}{d z^{\varpi}} .
$$

In ähnlicher Art findet man zweitens für ein grades $p$ von der Form $p=2 \widetilde{\omega}+2$ durch wiederholte Differentiation aus $\left(1-2 r z+r^{2}\right)^{-\frac{2}{2}}$, mit Einführung der Kugelfunction

den Ausdruck

$$
P^{n}(z)=I^{n}[2, z]
$$

$$
2^{\varpi} I^{n}[2 \widetilde{\omega}+2, z]=\frac{d^{\varpi} P^{n+\varpi}(z)}{d z^{\Phi}} .
$$


Man weiss (Handb. $\$ .5$ und 36), dass $P$ und $C$ sich durch die Formeln

$$
\begin{aligned}
& P^{n}(z)=\frac{1}{2^{n} \Pi n} \frac{d^{n}\left(z^{2}-1\right)^{n}}{d z^{n}}, \\
& C^{n}(z)=\frac{\sqrt{z^{2}-1}}{n \cdot 2^{n-1} \Pi \frac{2 n-1}{2}} \frac{d^{n}\left(z^{2}-1\right)^{\frac{2 n-1}{2}}}{d z^{n}}
\end{aligned}
$$

darstellen lassen; setzt man diese Werthe in die vorstehenden Formeln ein, so ergiebt sich als Ausdruck von $I^{n}[p, z]$, resp. für grade und ungrade $p$ :

$$
\begin{aligned}
& I^{n}[2 \widetilde{\omega}+2, z]=\frac{1}{2^{n+2 \varpi} \Pi(n+\varpi)} \frac{d^{n+2 \varpi}\left(z^{2}-1\right)^{n+\varpi}}{d z^{n+2 \varpi}}, \\
& I^{n}[2 \widetilde{\omega}+1, z]=\frac{1}{2^{n+2 \varpi-1}(n+\varpi) \Pi \frac{2 n+2 \varpi-1}{2}} \frac{d^{\varpi}}{d z^{\varpi}} \cdot\left[\sqrt{z^{2}-1} d^{n+\varpi} \cdot\left(z^{2}-1\right)^{\frac{2 n+2 \varpi-1}{2}}\right] .
\end{aligned}
$$

§. 10. Zu grösserer Bequemlichkeit ist dieser Arbeit am Schlusse eine Sammlung von Formeln hinzugefügt worden. Taf. I., II. und III. enthalten solche, die wir hier bereits abgeleitet haben, oder die, wie einige Ausdrücke in Taf. II., sich unmittelbar ergeben, auch die Definition der $I^{n}$ und ihre Beziehung zu den $I_{\nu}^{n}$. Ferner giebt Taf. IV. einige bekannte Ausdrücke, welc̀che unten gebraucht werden; Taf. VI. enthält Formeln, welche in der Arbeit „über einige bestimmte Integrale" im $61^{\text {sten }}$ Bande dieses Journals abgeleitet wurden, oder sogleich daraus folgen. Die Taf. VII. und VIII. enthalten Beziehungen, die erst in den folgenden Paragraphen erörtert werden sollen. Taf. V. endlich zeigt eine merkwürdige Formel von Jacobi (No. 32, \$. 43 im Handb.) und eine ihr entsprechende neue, die sich durch Verbindung von $\$$. 7 und 9 ergeben.

Um diese abzuleiten, hat man nur die Werthe von $I_{0}^{n}$ aus $\$ .7$ und $\$ .9$ einander gleich zu setzen. Für grade $p$ erhält man dann die erste Formel in Taf. V., für ungrade $p$ die zweite. Bei der Reduction hat man die einfachen Werthe zu beachten, welche nach der zweiten Formel in Taf. IV.

$$
\Pi(n+\widetilde{\omega}) \Pi \frac{2 n+2 \varpi-1}{2} ; \quad \Pi(n+\widetilde{\omega}-1) \Pi \frac{2 n+2 \varpi-1}{2}
$$

annehmen, und dann für $n+\widetilde{\omega}$ einen Buchstaben $m$ zu setzen.

\$. 11. Die bekanntesten Eigenschaften der Kugelfunctionen, welche sich bei den $I$ wiederfinden, übertragen wir nun auf die letzteren. Wir beginnen mit dem Hülfssatze, dass

$$
\int_{-1}^{+1} I_{v}^{m}[p, z] I_{v}^{n}[p, z]\left(z^{2}-1\right)^{\frac{t}{(p-2)}} d z
$$


verschwindet, weńn $m$ und $n$ verschieden sind. Dies zeigt sich sowohl aus der Differentialgleichung der $I$ im $\mathfrak{\$} .4$, als auch durch folgendes Verfahren, durch welches man zugleich den Werth des Integrals für $m=n$, der nicht verschwindet, bequem findet.

Zunächst ist klar, dass man nur nöthig hat das Integral für $\nu=0$ aufzusuchen; in der That wird nach Taf. II. der obige Ausdruck gleich

$$
\int_{-1}^{\cdot 1} I_{0}^{m-\nu}[p+2 \nu, z] I_{0}^{n-\nu}[p+2 \nu, z]\left(z^{2}-1\right)^{\frac{2 \nu+p-2}{2}} d z,
$$

also gleich dem Werthe des gegebenen, wenn man in ihm $\nu=0$ setzt, und nur $m, n, p$ resp. mit $m-\nu, n-v, p+2 \nu$ vertauscht.

Ist nun $\nu=0$, so unterscheiden wir zwei Fälle; es kann $p$ grade oder ungrade sein. Wir wollen nur den letzteren hier behandeln, da der erstere der einfachere, und wesentlich nichts Anderes als ein bekannter Satz für die Kugelfunctionen ist (Handb. \$. 52).

Es sei also $p=2 \widetilde{\omega}+1$, und ferner $m<n$. Nun ist nach $\$ .7$

$$
\left(z^{2}-1\right)^{\frac{p-2}{2}} I_{0}^{n}[p, z]=\frac{\Pi(n+p-2)}{\Pi(2 n+p-2)} \frac{d^{n}\left(z^{2}-1\right)^{\frac{2 n+2 \omega-1}{2}}}{d z^{n}} ;
$$

berücksichtigt man, dass $I^{m}[p, z]$ vom $m^{\text {ten }}$ Grade nach $z$, und dass $m<n$ ist, so wird unser Integral, wenn man $n$ mal durch Theile integrirt, Null geben und erst dann eine nicht verschwindende Grösse, wenn man $m=n$ setzt. Diese Grösse ist dann

$$
(-1)^{\frac{2 \omega-1}{2}} \frac{\Pi n \Pi(n+p-2)}{\Pi(2 n+p-2)} \int_{0}^{\pi} \sin ^{2 n+2 \pi} \varphi d \varphi
$$

oder nach Taf. IV.

$$
\frac{(-1)^{\frac{p-2}{2} \pi}}{2^{2 n+p-1}}(2 n+p-1) \frac{\Pi n \Pi(n+p-2)}{\left(\Pi \frac{2 n+p-1}{2}\right)^{2}}
$$

Für grade $p$ hätte man denselben Ausdruck für unser Integral erhalten; aus dem gewonnenen Resultate ist die Taf. VIl. gebildet.

\$. 12. Die Formeln des $\$ .11$ benutzen wir, um $\left(x+z \sqrt{x^{2}-1}\right)^{n}$, wenn $n$ ganz ist, in eine Reihe zu entwickeln, welche nach Functionen $I^{m}[p, z]$ mit veränderlichem $m$ aber festgehaltenem $p$ fortschreitet, d. h. in eine Reihe

(a.) $\quad\left(x+z \sqrt{x^{2}-1}\right)^{n}=\sum_{m=0}^{m=n} u_{m} I^{m}[p, z]$ 
wenn die $u$ von $z$ unabhängige Werthe, die natürlich noch von $x$ abhängig sind, vorstellen. Zunächst ist die Möglichkeit einer solchen Entwickelung nach $I^{m}$, weil sie ganze Functionen $m^{\text {ten }}$ Grades sind, klar; unsere Aufgabe besteht nur darin, die $u$ zu bestimmen.

Ist $p$ eine ungrade Zahl $2 \widetilde{\omega}+1$, so leitet man die gesuchte Formel durch blosse Differentiation nach $z$ aus der bekannten für $p=1$ geltenden (Handb. \$. 43, No. 33, a) ab:

$$
\frac{2^{n}}{\Pi 2 n}\left(x+\cos \psi \sqrt{x^{2}-1}\right)^{n}=\frac{P_{0}^{n}(x)}{\Pi n \Pi n}+2 \sum_{m=1}^{m=n} \frac{P_{m}^{n}(x) \cos m \psi}{\Pi(n+m) \Pi(n-m)} .
$$

Setzt man hier $z$ für $\cos \psi$ und drückt die Functionen $P$ und die Cosinus der Vielfachen von $\psi$ durch die allgemeineren Zeichen $I$ aus (Taf. I. und II.), so lautet die vorstehende Formel

$$
\frac{2^{n}}{\Pi 2 n}\left(x+z \sqrt{x^{2}-1}\right)^{n}=\sqrt{ } \pi \sum_{m=0}^{m=n} \frac{m I_{m}^{n}[2, x] I^{m}[1, z]}{\Pi(n+m) \Pi(n-m)} .
$$

Man differentiire diese $\widetilde{\omega}$ mal nach $z$, nachdem man vorher $n+\widetilde{\omega}$ für $n$ gesetzt hat, dividire durch $\left(\sqrt{x^{2}-1}\right)^{\varpi}$ und benutze Taf. III. und II.; dann entsteht die fül ungrade $p$ geltende Formel

$$
\left(x+z \sqrt{x^{2}-1}\right)^{n}=2^{n+p-2} \Pi n \Pi \frac{2 n+p-2}{2} \sum_{m=0}^{m=n} \frac{2 m+p-1}{\Pi(n-m) \Pi(n+m+p-1)} I_{m}^{n}[p+1, x] I^{m}[p, z] .
$$

\$. 13. Dieselbe Formel gilt für grade $p$, wie sich durch eine andere Methode zeigen lässt, die etwas mehr Rechnung als die frühere erfordert, die übrigens auch für ungrade $p$ hätte angewandt werden können.

Es sei also $p=2 \widetilde{\omega}+2$; wir multipliciren $(a$.$) im \$ .12 \mathrm{mit}$

$$
I^{m}[p, z]\left(z^{2}-1\right)^{\frac{1}{2}(p-2)} d z
$$

und integriren nach $z$ von -1 bis 1 . Dadurch entsteht aus der rechten Seite von $(a$.$) , nach Taf. VII., u_{m} c^{m}[p]$.

Die linke Seite von (a.) liefert nach Taf. III.

$$
\frac{1}{2^{m+2} \Pi \Pi(m+\varpi)} \int_{-1}^{1}\left(x+z \sqrt{x^{2}-1}\right)^{n}\left(z^{2}-1\right)^{\varpi} \frac{d^{m+2 \pi}\left(z^{2}-1\right)^{m+\varpi}}{d z^{m+2} \bar{\omega}} d z
$$

also nach Taf. $\mathrm{V}$.

$$
\frac{1}{2^{m+2 \varpi}} \frac{\Pi(m+2 \varpi)}{\Pi m \Pi(m+\varpi)} \int_{-1}^{1}\left(x+z \sqrt{\left.x^{2}-1\right)^{n}} \frac{d^{m}\left(z^{2}-1\right)^{m+\varpi}}{d z^{m}} d z .\right.
$$

Integrirt man $m$ mal durch Theile und setzt dann unter dem Integrale $z=\cos \psi$, so entsteht

$$
\frac{(-1)^{\varpi}}{2^{m+2 \varpi}} \frac{\Pi n \Pi(m+2 \varpi)\left(x^{2}-1\right)^{\frac{1}{2} m}}{\Pi(n-m) \Pi(m+\varpi) \Pi m} \int_{0}^{\pi}\left(x+\cos \psi \sqrt{x^{2}-1}\right)^{n-m} \sin ^{2 m+2 \varpi+1} \psi d \psi,
$$


und nach Taf. VI.

$$
(-1)^{\varpi} 2^{n+1} \frac{\Pi n \Pi(n+\varpi) \Pi(m+2 \varpi)}{\Pi m \Pi(n-m) \Pi(n+m+2 \varpi+1)}\left(\sqrt{x^{2}-1}\right)^{m} \mathfrak{\Im}_{0}^{n-m}[2 m+p+1, x] .
$$

Achtet man darauf, dass die beiden letzten, $x$ enthaltenden Factoren nach Taf. II. nichts Anderes als $I_{m}^{n}[p+1, x]$ sind, so erhält man nach Division mit $c^{m}[p]$ den Werth für $u_{m}$. Es ist nicht erforderlich ihn hier noch besonders aufzuführen, da er in der That, wie oben behauptet wurde, mit dem Factor von $I^{m}[p, z]$ in der Formel am Schlusse des $\$$. 12 übereinstimmt.

\$. 14. Setzt man

$$
\cos \gamma_{1}=\cos \eta_{1} \cos \psi_{1}+\sin \eta_{1} \sin \psi_{1} \cos \gamma_{2},
$$

so lässt sich bekanntlich für $p=2$ die Function $I^{n}\left[p, \cos \gamma_{1}\right]$, d. h. $P^{n}\left(\cos \gamma_{1}\right)$ in eine elegante, nach Cosinus der Vielfachen von $\gamma_{2}$ fortschreitende Reihe entwickeln. Eine ähnliche Reihe existirt für alle $p$, die grösser als 1 sind; wir leiten sie zunächst für grade $p$ durch Differentiation aus der bekannten für $p=2$ geltenden ab, also durch eine Methode, die man aus $\$ .12$ schon kennt.

Die erwähnte Laplacesche Formel heisst in unseren Zeichen (m. vergl. Handb. \$. 67, No. 49)

$$
I^{n}[2, z]=\sum_{m=0}^{m=n} \frac{(-1)^{m}}{\sqrt{ } \pi} \frac{m .4^{n}\left(\Pi \frac{2 n-1}{2}\right)^{2}}{\Pi(n+m) \Pi(n-m)} I_{m}^{n}[2, x] I_{m}^{n}\left[2, x_{1}\right] I^{m}[1, y],
$$

wenn man

$$
z=x x_{1}-y \sqrt{x^{2}-1} \sqrt{x_{1}^{2}-1}
$$

macht. Setzt man in derselben $n+\widetilde{\omega}$ für $n$, differentiirt $\widetilde{\omega}$ mal nach $y$, dividirt darauf durch $\left(\sqrt{x^{2}-1} \sqrt{x_{1}^{2}-1}\right)^{\varpi}$, und reducirt durch Taf. I-III., so entsteht der zunächst für grade $p$ geltende Ausdruck, in dem man wieder $z=\cos \gamma_{1}$, $x=\cos \eta_{1}, x_{1}=\cos \psi_{1}, y=\cos \gamma_{2}$ gesetzt hat,

$$
\begin{gathered}
I^{n}\left[p, \cos \gamma_{1}\right]=\sum_{m=0}^{m=n} k_{m}^{n}[p] I_{m}^{n}\left[p, \cos \eta_{1}\right] I_{m}^{n}\left[p, \cos \psi_{1}\right] I^{m}\left[p-1, \cos \gamma_{2}\right], \\
k_{m}^{n}[p]=(-1)^{m}(2 m+p-2) \frac{2^{2 n+p-3}}{\sqrt{ } \pi} \frac{\left(\Pi \frac{2 n+p-3}{2}\right)^{2}}{\Pi(n-m) \Pi(n+m+p-2)} .
\end{gathered}
$$

Wir machen noch auf den einfachen Zusammenhang, der $k$ mit den $c$ in Taf. VII. aufmerksam. Man hat nämlich offenbar

$$
k_{m}^{n}[p] c_{m}^{n}[p]=(-1)^{\frac{1}{(2 p-2)}} \frac{2 n+p-2}{2 n+p-1} \sqrt{ } \pi
$$


§. 15. Ehe wir zeigen, dass die vorstehendè Reihenentwickelung auch für ungrade $p$ gilt, bemerken wir, dass, sobald sie gilt, also zunächst für grade $p$, man auch hat

$$
\int_{0}^{\pi} I^{n}\left[p, \cos \gamma_{1}\right] \sin ^{p-2} \gamma_{2} d \gamma_{2}=2^{p-2} \gamma \pi \frac{\Pi(n) \Pi \frac{p-3}{2}}{\Pi(n+p-2)} I^{n}\left[p, \cos \eta_{1}\right] I^{n}\left[p, \cos \psi_{1}\right] .
$$

Für $p=2$ ist dies der bekannte Satz von Legendre im Handb. $\$$. 67, S. 176.

Man beweist diesen Satz auf der Stelle, indem man die unter dem Integrale befindliche Function $I^{n}\left[p, \cos \gamma_{1}\right]$ nach Anleitung des $\$ .14$ in eine nach $I^{m}\left[p-1, \cos \gamma_{2}\right]$, mit veränderlichem $m$ fortschreitende Reihe entwickelt, darauf mit $I^{0}\left[p-1, \cos \gamma_{2}\right]$, also einer Constanten, und mit $\sin ^{p-2} \gamma_{2} d \gamma_{2}$ multiplicirt, und nach $\gamma_{2}$ von 0 bis $\pi$ integrirt. Die rechte Seite reducirt sich dann, nach Taf. VII., auf das einzige Glied

$$
k_{0}^{n}[p] I_{0}^{n}\left[p, \cos \eta_{1}\right] I_{0}^{n}\left[p, \cos \psi_{1}\right] \int_{0}^{\top \pi}\left(I^{0}\left[p-1, \cos \gamma_{2}\right]\right)^{2} \sin ^{p-2} \gamma_{2} d \gamma_{2}
$$

das in demselben vorkommende Integral ist, bis auf das Vorzeichen, $c^{0}[p-1]$.

Anmerkung. Wegen einer Anwendung in dem dritten Abschnitte \$. 21 mag hier erwähnt werden, dass zur Ableitung des Satzes es nicht erforderlich ist, die Reihe des $\mathfrak{S}$. 14 vollständig zu kennen; eine ungefähre Kenntniss reicht hin. Dass $I^{n}\left[p, \cos \gamma_{1}\right]$ in eine nach $I^{m}\left[p-1, \cos \gamma_{2}\right]$ fortschreitende Reihe entwickelt werden kann, ist ohne Weiteres klar; zur Ableitung unseres Satzes genügt es schon, wenn man weiss, dass das $0^{\text {te }}$ Glied (d. h. dasjenige, welches $m=0$ entspricht) von der Form

$$
v I^{n}\left[p, \cos \eta_{1}\right] I^{0}\left[p-1, \cos \gamma_{2}\right]
$$

ist, wo $v$ kein $\eta_{1}$ und $\gamma_{2}$, also nur noch $\psi_{1}$ und Constante enthält. Unser Integral, welches an der Spitze dieses Paragraphen steht, wird nun, da man aus Taf. VII. weiss, dass nur das $0^{\text {te }}$ Glied der Reihe in Frage kommt,

wenn man setzt

$$
a v I^{n}\left[p, \cos \eta_{1}\right]
$$

$$
a=\int_{0}^{\pi} I^{0}[p-1, \cos \gamma] \sin ^{p-2} \gamma d \gamma .
$$

Macht man $\eta_{\mathrm{f}}=0$, so wird demnach

$$
\text { - } I_{n}^{n}\left[p, \cos \psi_{1}\right] \int_{0}^{\pi} \sin ^{p-2} \gamma d \gamma=a v I^{n}[p, 1],
$$

woraus sich mit Hülfe von Taf. I. sofort $a v$ ergiebt. 
\$. 16. Indem wir nun die Formel des $\$ .14$ für ein ungrades $p$, von der Form $p=2 \widetilde{\omega}+3$ ableiten, wollen wir zur Bequemlichkeit des Druckes die Anzahl der Indices vermindern, und als Argument von $I^{n}$

$$
\cos \gamma=\cos \eta \cos \psi+\sin \eta \sin \psi \cos \gamma_{\iota}
$$

wählen; es wird ferner eine Grösse

$$
\cos \delta=\cos \gamma_{1} \cos \theta_{1}+\sin \gamma_{1} \sin \theta_{1} \cos \theta
$$

eingeführt.

In der Formel des $\$$. 9 unsrer Arbeit über einige bestimmte Integrale (Band 61, pag. 356 dieses Journals) setzen wir $p$ für $n$ und

$$
\begin{gathered}
a=\cos \eta-r \cos \psi, \\
a_{1}=\sin \eta-r \sin \psi \cos \gamma_{1}, \\
a_{2}=-r \sin \psi \sin \gamma_{1}, \\
a_{3}=a_{4}=\text { etc. }=a_{p}=0 ;
\end{gathered}
$$

dann wird aus derselben die Gleichung erhalten

$$
=b \frac{\Pi \frac{p-2}{2}}{2 \pi^{\frac{p}{2}}} \int_{0}^{\pi} d \theta_{1} \int_{0}^{2 \pi} \frac{1}{\left(1-2 r \cos \gamma+r^{2}\right)^{\frac{p-1}{2}}}
$$

wo $b$ ein $p$-3faches Integral

$$
\int^{p-3} \sin \theta_{3} \ldots \sin \theta_{p-2} d \theta_{3} \ldots d \theta_{p-1}=\frac{2 \pi^{\frac{p-2}{2}}}{\Pi \frac{p-4}{2}}
$$

bezeichnet. Entwickelt man nun auf der linken und rechten Seite nach aufsteigenden Potenzen von $r$, so findet man nach Taf. I.

$$
I^{n}[p, \cos \gamma]=\frac{\Pi(n+p-2)}{2^{p-2} \pi \Pi n \Pi \frac{p-4}{2}} \int_{0}^{\pi} \frac{M \sin ^{p-2} \theta_{1} d \theta_{1}}{\left(\cos \eta+i \sin \eta \cos \theta_{1}\right)^{n+p-1}},
$$

wenn man zur Abkürzung setzt

$$
M=\int_{0}^{\pi}(\cos \psi+i \sin \psi \cos \delta)^{n} \sin ^{p-3} \theta d \theta
$$

Zunächst kann $M$ von dem Integralzeichen befreit werden; macht man nämlich

$$
(\cos \psi+i \sin \psi \cos \delta)^{n}=\sum_{m=0}^{m=n} u_{m} I^{m}[p-1, \cos \delta],
$$

Journal für Mathematik Bd. LXII. Heft 2. 
wobei der Werth, den dann $u_{m}$ hat, sogleich aus der ersten Formel in Taf. VIII. ersichtlich ist, so wird

$$
M=\sum_{m=0}^{m=n} u_{m} \int_{0}^{\pi} I^{m}[p-1, \cos \delta] \sin ^{p-3} \theta d \theta
$$

Der Werth der Integrale, welche in dieser Reihe auftreten, ist aus $\$ .15$ bekannt, da $p-1=2 \widetilde{\omega}+2$ eine grade Zahl bezeichnet; die Reihe für $M$, die man so erhält, setzt man in den Ausdruck für $I^{n}[p, \cos \gamma]$, welcher sich dann in die Summe von Gliedern verwandelt, deren $m^{\text {tes }}$

$$
\frac{2 m+p-2}{\sqrt{\pi}} \cdot 2^{n+p-4} \frac{\Pi m \Pi(n+p-2) \Pi \frac{2 n+p-3}{2}}{\Pi(n-m) \Pi(m+p-3) \Pi(m+n+p-2)} v_{m} I_{m}^{n}[p, \cos \psi] I^{m}\left[p-1, \cos \gamma_{1}\right]
$$

ist, und zwar hat man die Summe von $m=0$ bis $m=n$ zu nehmen. Zur Abkürzung ist hier gesetzt:

$$
v_{m}=\int_{0}^{\pi} \frac{I^{m}[p-1, \cos \theta] \sin ^{p-2} \theta d \theta}{(\cos \eta+i \sin \eta \cos \theta)^{n+p-1}} .
$$

Es bleibt noch übrig, das letzte Integral auszuführen. Da $p-3$ grade, $=2 \widetilde{\omega}$ ist, so wird, wenn $\operatorname{man} \cos \theta=z$ macht,

$$
\sin ^{p-3} \theta . I^{m}[p-1, \cos \theta]=(-1)^{\varpi} \cdot \frac{\left(z^{2}-1\right)^{\varpi}}{2^{m+2} \Pi \Pi(m+\varpi)} \frac{d^{m+2}\left(z^{2}-1\right)^{m+\varpi}}{d z^{m+2} \Phi},
$$

und nach Taf. V.

$$
=\frac{(-1)^{\varpi}}{2^{m+2 \Phi}} \frac{\Pi(m+2 \varpi)}{\Pi m \Pi(m+\varpi)} \frac{d^{m}\left(z^{2}-1\right)^{m+\pi}}{d z^{m}} .
$$

Setzt man diesen Werth in $v_{m}$ ein und integrirt $m$ mal durch Theile, führt darauf wieder $\cos \theta$ statt $z$ ein, so wird

$$
v_{m}=\frac{(-i \sin \eta)^{m}}{2^{m+2}} \frac{\Pi(m+2 \varpi) \Pi(m+n+p-2)}{\Pi m \Pi(m+\varpi) \Pi(n+p-2)} \int_{0}^{\pi} \frac{\sin ^{2 m+p-2} \theta d \theta}{(\cos \eta+i \sin \eta \cos \theta)^{m+n+p-1}} .
$$

Den Werth dieses Integrales kennt man aus Taf. VI; berücksichtigt man, dass nach Taf. II

$$
(i \sin \eta)^{m} I_{0}^{n-m}[p+2 m, \cos \eta]=I_{m}^{n}[p, \cos \eta],
$$

so erhält man den Ausdruck von $v_{m}$ in der schliesslichen Form; setzt man ihn ein, so entsteht dieselbe Formel für $I^{n}[p, \cos \gamma]$ bei ungradem $p$, welche wir im $\$$. 14 für grade $p$ fanden.

Der Beweis, welchen wir hier lieferten, ist eine unmittelbare Uebertragung des von Jacobi für die Laplacesche Reihe gegebenen; die einzige Schwierigkeit bei der Uebertragung bestand in der V́erallgemeinerung der 
Jacobischen Formel

$$
\frac{1}{2 \pi} \int_{0}^{2 \pi} \frac{d \theta}{a+a_{1} i \cos \theta+a_{2} i \sin \theta}=\frac{1}{\sqrt{a^{2}+a_{1}^{2}+a_{2}^{2}}},
$$

welche man in unserer früheren Arbeit über einige bestimmte Integrale kennen lernte.

\$. 17. Wir stellen nun die Bezeichnung des $\$ .14$ wieder her. Die dort gewonnene Formel, welche jetzt für grade und ungrade $p$ gilt, setzt nur voraus, dass die ganze Zahl $p$ nicht unter 2 herabsinkt. Ist $p>2$, so kann man dieselbe Fundamentalgleichung wieder anwenden, um die in ihr vorkommenden Grössen $I^{m}\left[p-1, \cos \gamma_{2}\right]$ noch weiter in ähnliche Reihen zu entwickeln. Ist $p>3$, so kann dasselbe Verfahren noch einmal wiederholt werden, u. s. f. Aus dem einfachen Satze des $\$$. 14 hat man demnach sogleich den allgemeineren:

Ist

$$
\begin{aligned}
& \cos \gamma_{1}=\cos \eta_{1} \cos \psi_{1}+\sin \eta_{1} \sin \psi_{1} \cos \gamma_{2}, \\
& \cos \gamma_{2}=\cos \eta_{2} \cos \psi_{2}+\sin \eta_{2} \sin \psi_{2} \cos \gamma_{3}^{\prime}, \\
& \cdot \cdot \cdot \cdot \cdot \cdot \cdot \cdot \cdot \cdot \cdot \cdot \cdot \cdot \cdot \cdot \cdot \\
& \cos \gamma_{p-1}=\cos \eta_{p-1} \cos \psi_{p-1}+\sin \eta_{p-1} \sin \psi_{p-1} \cos \gamma_{p},
\end{aligned}
$$

so entsteht $I^{n}\left[p, \cos \gamma_{1}\right]$ aus dem Producte

wenn gesetzt ist

$$
K . H . \Psi . I^{n_{p-1}}\left[1, \cos \gamma_{p}\right]
$$

$$
\begin{aligned}
& K=k_{n_{1}}^{n}[p] k_{n_{2}}^{n_{1}}[p-1] \ldots k_{n_{p-1}}^{n_{p-2}}[2], \\
& H=I_{n_{1}}^{n}\left[p, \cos \eta_{1}\right] I_{n_{2}}^{n_{1}}\left[p-1, \cos \eta_{2}\right] \ldots I_{n_{p-1}}^{n_{p-2}}\left[2, \cos \eta_{p-1}\right], \\
& \Psi=I_{n_{1}}^{n}\left[p, \cos \psi_{1}\right] I_{n_{2}}^{n_{1}}\left[p-1, \cos \psi_{2}\right] \ldots I_{n_{p-1}}^{n_{p-2}}\left[2, \cos \psi_{p-1}\right],
\end{aligned}
$$

durch Summation über alle nicht negativen ganzzahligen Werthe (incl. Null) von $n_{1}, n_{2}$, etc., $n_{p-1}$, für welche

$$
n-n_{1}, \quad n_{1}-n_{2}, \quad \text { etc., } n_{p-2}-n_{p-1}
$$

nicht negativ werden. Die Anzahl der Glieder, aus denen $I^{n}\left[p, \cos \gamma_{1}\right]$ besteht, ist also

$$
\frac{(n+1)(n+2) \ldots(n+p-1)}{1.2 \ldots(p-1)}
$$


§. 18. Nennt man die Kugelfunction

$$
P^{n}\left(\cos \psi \cos \theta+\sin \psi \sin \theta \cos \left(\theta_{1}-\psi_{1}\right)\right)
$$

zur Abkürzung $\boldsymbol{P}$, dieselbe Function mit den Argumenten

$$
\begin{aligned}
& \cos \eta \cos \theta+\sin \eta \sin \theta \cos \left(\theta_{1}-\eta_{1}\right), \\
& \cos \eta \cos \psi+\sin \eta \sin \psi \cos \left(\psi_{1}-\eta_{1}\right)
\end{aligned}
$$

resp. $P_{1}$ und $P_{2}$, so sagt ein bekannter Satz, dass

$$
P_{2}=\frac{2 n+1}{4 \pi} \int_{0}^{\pi} d \theta \sin \theta \int_{11}^{2 \pi} P P_{1} d \theta_{1}
$$

ist. Wir können dies auch so aussprechen: Sind die $a$ und $b$ solche Constante, dass

$$
a^{2}+a_{1}^{2}+a_{2}^{2}=1, \quad b^{2}+b_{1}^{2}+b_{2}^{2}=1,
$$

und bezeichnet man die Functionen $P^{n}$ mit den Argumenten

$$
a x+a_{1} x_{1}+a_{2} x_{2}, \quad b x+b_{1} x_{1}+b_{2} x_{2}, \quad a b+a_{1} b_{1}+a_{2} b_{2}
$$

resp. durch $P, P_{1}, P_{2}$, so ist

$$
P_{2}=\frac{2 n+1}{4 \pi} \int P P_{1} \frac{d x d x_{1}}{x_{2}}
$$

wenn das Doppelintegral rechts sich über alle Werthe $x$ und $x_{1}$ erstreckt, für die $x^{2}+x_{1}^{2}+x_{2}^{2}=1$ ist.

Die Verallgemeinerung dieser Formel giebt einen entsprechenden Satz, den wir hier beweisen wollen: Sind die Constanten $a$ und $b$ so beschaffen, dass

$$
a^{2}+a_{1}^{2}+\text { etc. }+a_{p}^{2}=1, \quad b^{2}+b_{1}^{2}+\text { etc. }+b_{p}^{2}=1,
$$

bezeichnet man ferner die drei Functionen $p^{\text {ter }}$ Ordnung $I^{n}[p, z]$, deren $\operatorname{Ar}$ gumente $z$ resp.

$$
\begin{aligned}
& a x+a_{1} x_{1}+\text { etc. }+a_{p} x_{p}, \\
& b x+b_{1} x_{1}+\text { etc. }+b_{p} x_{p}, \\
& a b+a_{1} b_{1}+\text { etc. }+a_{p} b_{p}=\cos \gamma
\end{aligned}
$$

sind, durch $I, I_{1}, I_{2}$, so wird

$$
\int I I_{1} \frac{d x d x_{1} \ldots d x_{p-1}}{x_{p}}=\frac{4 \pi^{\frac{p}{2}}}{2 n+p-1} I_{2},
$$

wenn das $p$ fache Integral auf der Linken sich über alle Werthe $x, x_{1}, \ldots, x_{p-1}$ erstreckt, für welche die Gleichung stattfindet

$$
x^{2}+x_{1}^{2}+\cdots+x_{p}^{2}=1 \text {. }
$$

Zum Beweise kann man sich des Satzes im \$. 11 der Arbeit über einige bestimmte Integrale bedienen; setzt man dort $p+1$ statt $n$, so sieht man, 
dass das vielfache Integral, welches wir hier betrachten, sich auf das Doppelintegral

$\frac{2 \pi^{\frac{p-1}{2}}}{\Pi \frac{p-3}{2}} \int_{0}^{\pi} I^{n}[p, \cos \varphi] \sin ^{p-1} \varphi d \varphi \int_{0}^{2 \pi} I^{n}\left[p, \cos \varphi \cos \gamma+\sin \varphi \sin \gamma \cos \varphi_{1}\right] \sin ^{p-2} \varphi_{1} d \psi_{1}$ reducirt. Nach $\mathfrak{S}$. 15 lässt sich das innere Integral ausführen, und giebt zunächst einen constanten Factor, der mit der Constanten vor dem Doppelintegral verbunden

$$
\frac{1}{c^{n}[p]} \cdot \frac{4 \pi^{\frac{p}{2}}}{2 n+p-1}
$$

liefert, dann $I^{n}[p, \cos \gamma]$, und endlich $I^{n}[p \cos \varphi]$, welches unter das äussere Integral tritt und dies zu $c^{n}[p]$ macht.

Man hätte denselben Satz auch mit Hülfe der einfachen Beziehung beweisen können, welche nach der Bemerkung am Schlusse des $\mathbb{\$} .14$ zwischen $c$ und $k$ besteht.

\section{Dritter Abschnitt.}

\$. 19. Der Ausdruck

$$
T=\frac{1}{\left(\sqrt{(\xi-a)^{2}+\left(\xi_{1}-a_{t}\right)^{2}+\cdots+\left(\xi_{p}-a_{p}\right)^{2}}\right)^{p-1}}
$$

genügt, wie man weiss, der Differentialgleichung

$$
\text { (5.) } \frac{\partial^{2} \boldsymbol{T}}{\partial \xi^{2}}+\frac{\partial^{2} \boldsymbol{T}}{\partial \xi_{1}^{2}}+\cdots+\frac{\partial^{2} \boldsymbol{T}}{\partial \xi_{p}^{2}}=\mathbf{0}
$$

wenn die $a$ irgend welche Constante bezeichnen; zur grösseren Bequemlichkeit im Ausdrucke nehmen wir an, es sei

$$
a^{2}+a_{1}^{2}+\cdots+a_{p}^{2}=1 \text {. }
$$

Wir schliessen ferner der Kürze halber den Fall $p=1$ aus, in welchem man bekanntlich statt einer Potenz für $\boldsymbol{T}$ einen Logarithmus zu nehmen hat. Setzt man

$$
\begin{aligned}
& \xi=r x, \quad \xi_{1}=r x_{1}, \quad \ldots, \quad \xi_{p}=r x_{p}, \\
& x=\cos \psi_{1}, \quad a=\cos \eta_{1} \text {; } \\
& x_{1}=\sin \psi_{1} \cos \psi_{2}, \quad a_{1}=\sin \eta_{1} \cos \eta_{2}, \\
& x_{2}=\sin \psi_{1} \sin \psi_{2} \cos \psi_{3}, \quad \text { etc. etc. } \\
& \dot{x}_{p-1}=\dot{\sin } \psi_{1} \sin \psi_{2} \ldots \cdot \sin \psi_{p-1} \cos \psi_{p} \text {, } \\
& x_{p}=\sin \psi_{1} \sin \psi_{2} \ldots \sin \psi_{p-1} \sin \psi_{p} \text {, }
\end{aligned}
$$


so wird die Differentialgleichung, der nun

$$
T=\frac{1}{\left(\sqrt{1-2 r \cos \gamma_{1}+r^{2}}\right)^{p-1}}
$$

genügt, durch Transformation von (5.) in die neuen Coordinaten gefunden. Es hat hier $\cos \gamma_{1}$ dieselbe Bedeutung wie im $\$$. 17, dessen Bezeichnung wir in diesem Abschnitte beibehalten; nur ist hier $\psi_{p}-\eta_{p}$ für $\gamma_{p}$ zu setzen. Die Ausführung der Rechnung, welche ich der Güte eines hiesigen Freundes verdanke, giebt folgende Gleichung für $\boldsymbol{T}$ :

$$
\mathbf{0}=\frac{\partial}{\partial \boldsymbol{r}} \cdot\left[\boldsymbol{\Delta} \frac{\partial \boldsymbol{T}}{\partial \boldsymbol{r}}\right]+\frac{\partial}{\partial \psi_{1}} \cdot\left[\frac{\Delta}{u_{1}} \cdot \frac{\partial \boldsymbol{T}}{\partial \psi_{1}}\right]+\cdots+\frac{\partial}{\partial \psi_{p}} \cdot\left[\frac{\Delta}{u_{p}} \cdot \frac{\partial \boldsymbol{T}}{\partial \psi_{p}}\right] .
$$

Hier ist zur Abkürzung gesetzt

$$
\begin{aligned}
& \Delta=r^{p} \sin ^{p-1} \psi_{1} \sin ^{p-2} \psi_{2} \ldots \sin \psi_{p-1}, \\
& u_{1}=r^{2} \\
& u_{2}=r^{2} \sin ^{2} \psi_{1}, \\
& \ldots \ldots \ldots \sin ^{2} \psi_{p-1} .
\end{aligned}
$$

Entwickelt man nun $\boldsymbol{T}$ nach aufsteigenden Potenzen von $r$, so genügt der Coefficient von $r^{n}$, also auch $I^{n}\left[p, \cos \gamma_{1}\right]$ der Gleichung

$$
\text { (6.) } \quad 0=n(n+p-1) I+\sum_{m=1}^{m=p} \frac{w_{m}}{\sin ^{p-m} \psi_{m}} \frac{\partial}{\partial \psi_{m}} \cdot\left[\sin ^{p-m} \psi_{m} \frac{\partial \boldsymbol{I}}{\partial \psi_{m}}\right] \text {, }
$$

in der gesetzt ist

$$
w_{1}=1, \quad \frac{1}{w_{m}}=\left(\sin \psi_{1} \sin \psi_{2} \ldots \sin \psi_{m-1}\right)^{2} .
$$

Im speciellen Falle $\eta_{1}=0$ wird $\gamma_{1}=\psi_{1}$, also unabhängig von $\psi_{2}$, etc., so dass $I^{n}\left[p, \cos \psi_{1}\right]$ der Gleichung genügt

$$
0=n(n+p-1) I+\frac{1}{\sin ^{p-1} \psi_{1}} \frac{\partial}{\partial \psi_{1}}\left[\sin ^{p-1} \psi_{1} \frac{\partial I}{\partial \psi_{1}}\right] \text {. }
$$

§. 20. Die Function $I^{n}\left[p, \cos \gamma_{1}\right]$ ist ganz und vom Grade $n$ nach $\cos \gamma_{1}$, also auch, wegen der Zusammensetzung von $\cos \gamma_{1}$ aus den $x$, ganz und vom Grade $n$ nach $x, x_{1}$, etc. Die einzelnen Potenzen von $\cos \gamma_{1}$, die in $I^{n}$ vorkommen, sind die $n^{\text {te }}, n-2^{\text {te }}$, etc., aber nicht die $n-1^{\text {te }}, n-3^{\text {te }}$, etc.; jede Potenz einzeln in die $x$ umgesetzt giebt eine homogene Function derselben, so dass also unser $I^{n}$ die Summe von homogenen Functionen $n^{\text {ten }}$, $n-2^{\text {ten, }}$, etc. Grades der $x$ ist oder, weil

$$
x^{2}+x_{1}^{2}+\text { etc. }+x_{p}^{2}=1,
$$

eine homogene Function der $x$ vom $n^{\text {ten }}$ Grade. 
Wir wollen nun die Form der allgemeinsten homogenen Function der $x$ vom $n^{\text {ten }}$ Grade suchen, welche der Gleichung (6.) geniigt; sie heisse $X^{(n)}$.

Eine ganze Function der Grössen $\cos \psi_{1}, \cos \psi_{2}$, etc.; $\sin \psi_{1}, \sin \psi_{2}$, etc., welche (6.) genügt, ist offenbar die Summe von Gliedern

$$
\Psi\left(L \cos \left(n_{p-1} \psi_{p}\right)+M \sin \left(n_{p-1} \psi_{p}\right)\right)
$$

wenn $L$ und $M$ willkürliche Constante vorstellen, und die Summation sich über die $n$ in derselben Art erstreckt wie im \$. 17. Diese Summe werde vorläufig $Z$ genannt; es wird sich zeigen, dass $Z$ mit $X^{(n)}$ übereinstimmt.

Da für $n_{p-1}=0$ das Glied von $Z$, welches den Sinus enthält, verschwindet, so wird man alle Glieder von $Z$ erhalten, wenn man bei der Summation in dem ersten Gliede von $Z$ (dem Gliede, welches die Cosinus enthält) allen $n$ die Werthe giebt, welche ihnen nach $\$ .17$ zukommen; in dem zweiten Gliede von $Z$ kann man aber den Werth $n_{p-1}=0$ fortlassen, also über $n_{1}, n_{2}$, etc. $n_{p-1}$ erst von 1 an bis $n$, natürlich wieder so summiren, dass

$$
n-n_{1}, \quad n_{1}-n_{2}, \quad \text { etc., } \quad n_{p-2}-n_{p-1}
$$

nicht negativ werden. Man sieht hieraus, dass in $Z$ im Ganzen

$$
\frac{(n+1)(n+2) \ldots(n+p-1)+n(n+1) \ldots(n+p-2)}{1.2 .3 \ldots(p-1)}=\sigma
$$

willkürliche Constante $L$ und $M$ eintreten; $\$$. 23 zeigt sich, dass diese $\sigma$ Constante sich nicht zu einer geringeren Zahl zusammenziehen.

\$. 21. Ehe wir weiter gehen, wollen wir in diesem Paragraphen über einige Sätze handeln, die im vorigen Abschnitte bereits abgeleitet sind; es wird hierbei klarer werden, wie die Uebertragung der Laplaceschen Methoden von dem Falle $p=2$ auf den allgemeinen sich zu der Uebertragung der Jacobischen, die man im zweiten Abschnitte kennen lernte, gestaltet.

Den Satz des $\$$. 11 über

$$
\int_{-1}^{1} I_{\nu}^{m}[p, z] I_{\nu}^{n}[p, z]\left(z^{2}-1\right)^{\frac{p-2}{2}} d z
$$

kann man auch aus der Differentialgleichung der $I_{v}^{n}$ im $\$$. 4 auf die bekannte Laplacesche Art vermittelst Integration durch Theile herleiten. Hier zeigt sich sofort, dass das Integral verschwindet, wenn $m$ und $n$ verschieden sind; sein Werth $c_{\nu}^{n}[p]$ in Taf. VII für $m=n$ lässt sich durch dieselbe Methode, indem man die Glieder genauer betrachtet, welche bei der Integration durch Theile vor die Integrale treten, aber nicht ohne Schwierigkeit ermitteln.

Nimmt man an, dass $I^{n}\left[p, \cos \gamma_{1}\right]$ die Form von $Z$ im vorigen Paragraphen besitzt, so lässt sich die Reihe des $\$ .17$ auch auf folgende Art auf- 
finden: Zunächst sieht man leicht ein, dass $I$ dann die Form haben muss:

$$
\Sigma L \Psi H I^{n_{p-1}}\left[1, \cos \left(\psi_{p}-\eta_{p}\right)\right]
$$

wenn $L$ wieder Constante bezeichnet, und die Summation in der früheren Art ausgeführt wird. Hieraus beweist man den Satz des \$. 15 auf die Art, welche man dort in der Anmerkung findet, und dann den Satz des $\$$. 18 nach der Methode, welche in jenem Paragraphen angegeben ist. Aus diesem und dem Satze des $\$$. 11 folgt nun unmittelbar

$$
(L)^{2} c_{n_{1}}^{n}[p] c_{n_{2}}^{n_{2}}[p-1] \cdots=\frac{4 \pi^{\frac{1}{2} p}}{2 n+p-1} L,
$$

also mit Berücksichtigung des einfachen Zusammenhanges der $c$ und $k$ (\$. 14) wird $L=K$, wie man es im $\$$. 17 findet, oder $L=0$. Erst eine besondere Untersuchung zeigt, dass kein $L$ verschwindet.

\$. 22. Wir kehren nun zu den Betrachtungen des $\$$. 20 zurück. Aus dem ersten Theile des Satzes im $\mathfrak{\$} .11$ folgt sogleich, dass

$$
\text { (a.) } \int \mathrm{YZ} \frac{d x d x_{1} \ldots d x_{p-1}}{x_{p}}
$$

verschwindet, wenn man die Integration über alle Werthe $x, x_{1}$, etc., $x_{p-1}$ ausdehnt, für welche $x^{2}+x_{1}^{2}+$ etc. $+x_{p}^{2}=1$ wird, und $Z$ unseren Werth im $\$$. 20 vorstellt, $Y$ aber eine Function derselben Art, welche zu einem Werthe $m$ gehört, der von $n$ verschieden ist. Das Integral verschwindet offenbar noch immer, wenn für $Y$ und $Z$ verschiedene Glieder aus dem Ausdrucke gesetzt werden, der im $\$$. 20 mit $Z$ bezeichnet wurde, und auch im Folgenden so heissen soll.

Nimmt man noch den zweiten Theil des Satzes im $\$ .11$ hinzu, so beweist man auch leicht, dass

$$
\text { (b.) } \int I^{n}\left[p, \cos \gamma_{1}\right] \bar{Z} \frac{d a d a_{1} \ldots d a_{p-1}}{a_{p}}=\frac{4 \pi^{\frac{1}{2} p}}{2 n+p-1} Z
$$

ist, wenn $\bar{Z}$ den Werth bezeichnet, welcher aus $\boldsymbol{Z}$ dadurch entsteht, dass man alle $\psi$ mit $\eta$ vertauscht $(\$ 19)$ oder alle $x$ mit $a$, und wenn man so integrirt, dass $a^{2}+a_{1}^{2}+$ etc. $+a_{p}^{2}$ gleich 1 wird. Einen speciellen Fall von $(b$. hat man bereits im $\mathfrak{S} .18$ kennen gelernt.

Da $I^{n}\left[p, \cos \gamma_{1}\right]$ eine homogene Function $n^{\text {ten }}$ Grades der $x$ ist, so wird nach (b.) auch $Z$ dieselbe Eigenschaft besitzen, indem $\bar{Z}$ nur Constante nach den $x$, nämlich die $a$ enthält, welche in $I^{n}[p, \cos \gamma]$ nur in ganzen Potenzen auftreten. Wir haben also das neue Resultat: 
Die im $\mathfrak{S} .20$ angegebene Grösse $\mathrm{Z}$ ist eine homogene Function $n^{\text {ten }}$ Grades der $x$.

\$. 23. Die $\sigma$ Grössen $L$ und $M$ in $Z$ sind dort mit eben so vielen Grössen $\Psi \cos \left(n_{p-1} \psi_{p}\right)$ und $\Psi \sin \left(n_{p-1} \psi_{p}\right)$, die für den Augenblick $u_{1}, u_{2}$, etc., $u_{\sigma}$ heissen sollen, multiplicirt, zwischen denen keine lineare homogene Gleichung mit constanten Coefficienten stattfinden kann.

Denn wäre $b_{1} u_{1}+b_{2} u_{2}+$ etc. $+b_{\sigma} u_{\sigma}=0$ eine solche Gleichung, in welcher die $b$ also numerische Constante vorstellen, so würde man vermittelst (a.) und (b.) im $\$$. 22, durch die ganz bekannte Methode der Coefficientenbestimmung finden, dass jedes $b$. Null sein muss.

Kann man nun, wie es pag. 138 geschieht, nachweisen, dass $X^{(n)}$, d. h. dass die allgemeinste Function $n^{\text {ten }}$ Grades der $x$, welche (6.) genügt, höchstens $\sigma$ Constante linear enthält, mit anderen Worten, dass $X^{(n)}$ die Form hat

$$
a_{1} v_{1}+a_{2} v_{2}+\text { etc. }+a_{\tau} v_{\tau}
$$

wo die $a$ willkürliche Constante, die $v$ bestimmte Functionen der $x$ sind und $\tau \leqq \sigma$ ist: so folgt hieraus bekanntlich. die Uebereinstimmung von $X^{(n)}$ und $Z$. Denn zunächst folgt, dass jede der $\sigma$ Grössen $u$ (s. oben) sich als lineare Verbindung der $v$ darstellen lässt, dass also z. B.

$$
u_{m}=a_{1}^{(m)} v_{1}+a_{2}^{(m)} v_{2}+\text { etc. }+a_{\tau}^{(m)} v_{\tau} ;
$$

aus dem Anfange dieses Paragraphen erhellt, dass zwischen den $\sigma$ rechten Seiten des Gleichungssystemes, welches man aus der obigen Gleichung für $m=1$, etc., $m=\sigma$ findet, keine lineare Gleichung besteht. Hieraus geht hervor, dass $\tau=\sigma$, dann aber, dass jedes $v$, also auch dass

$$
X^{(n)}=a_{1} v_{1}+a_{2} v_{2}+\text { etc. }+a_{\tau} v_{\tau}
$$

die Form hat

$$
X^{(n)}=b_{1} u_{1}+b_{2} u_{2}+\text { etc. }+b_{\sigma} u_{\sigma} .
$$

Es ist offenbar $r^{n} Z^{n}$ eine homogene Function $n^{\text {ten }}$ Grades der $\xi$, welche (5.) genügt, $r^{n} X^{(n)}$ die allgemeinste homogene derartige Function. Wird noch der oben erwähnte Punkt über die Anzahl der Constanten in $X^{(n)}$ und zugleich über die Art ihres Auftretens bewiesen, so hat man also den Satz:

Die allgemeinste homogene Function der $x$ vom $n^{\text {ten }}$ Grade, welche (6.) genuigt, ist

$$
X^{(n)}=\Sigma \Psi\left(L \cos \left(n_{p-1} \psi_{p}\right)+M \sin \left(n_{p-1} \psi_{p}\right)\right) ;
$$

die allgèmeinste homogene Function $n^{\text {ten }}$ Grades der $\xi$, welche (5.) genügt, ist 
$r^{n} X^{(n)}$. Es bedeuten hier $L$ und $M$ im Ganzen

$$
\sigma=(2 n+p-1) \frac{(n+1)(n+2) \ldots(n+p-2)}{1.2 .3 \ldots(p-1)}
$$

willkürliche Constanten; ferner ist

$$
\Psi=I_{n_{1}}^{n}\left[p, \cos \psi_{1}\right] I_{n_{2}}^{n_{1}}\left[p-1, \cos \psi_{2}\right] \ldots I_{n_{p-1}}^{n_{p-2}}\left[2, \cos \psi_{p-1}\right]
$$

und die Summation über alle nicht negativen Werthe $n_{1}, n_{2}$, etc., $n_{p} z u$ erstrecken, für welche

nicht negativ wird.

$$
n-n_{1}, \quad n_{1}-n_{2}, \quad \text { etc., } n_{p-1}-n_{p}
$$

Um nun schliesslich das Fehlende über die Anzahl der Constanten nachzutragen, welche $r^{n} X^{(n)}$ besitzen kann, bezeichnen wir

$$
\frac{\partial^{2} \boldsymbol{V}}{\partial \xi_{1}^{2}}+\frac{\partial^{2} V}{\partial \xi_{2}^{2}}+\text { etc. }+\frac{\partial^{2} V}{\partial \xi_{p}^{2}}
$$

mit $\Delta V$, suchen also die allgemeinste Form einer homogenen Function $n^{\text {ten }}$ Grades $V$ von den $\xi$, welche der Gleichung

$$
\text { (c.) } \frac{\partial^{2} V}{\partial \xi^{2}}+\Delta V=0
$$

genügt. Indem man $V$ nach Potenzen von $\xi$ ordnet, kann man setzen

$$
V=f_{n}+\xi f_{n-1}+\xi^{2} f_{n-2}+\text { etc. }+\xi^{n-1} f_{1}+\xi^{n} f_{10},
$$

wo $f_{0}, f_{1}$, etc., $f_{n}$ homogene Functionen resp. des Grades 0 , 1, etc., $n$ von $\xi_{1}, \xi_{2}$, etc., $\xi_{n}$ vorstellen. Die Gleichung (c.) verlangt dann, dass man habe

$$
\begin{gathered}
\Delta f_{n}+\xi \Delta f_{n-1}+\cdots+\xi^{n-3} \Delta f_{3}+\xi^{n-2} \Delta f_{2} \\
=1.2 . f_{n-2}+2.3 . \xi f_{n-3}+\cdots+(n-1)(n-2) \xi^{n-3} f_{1}+n(n-1) \xi^{n-2} f_{10},
\end{gathered}
$$

d. h. dass das System von Gleichungen erfüllt werde:

$$
\text { 1.2. } f_{n-2}=\Delta f_{n}, \quad 2.3 \cdot f_{n-3}=\Delta f_{n-1}, \quad \text { etc., } \quad n(n-1) f_{1}=\Delta f_{2} \text {. }
$$

Es sind also $f_{n}$ und $f_{n-1}$ ganz willkürliche, nur nach der Festsetzung homogene Functionen des Grades $n$ und $n-1$ von den $p$ Veränderlichen $\xi_{1}$, $\xi_{2}$, etc., $\xi_{p}$; die übrigen $f$ folgen aus diesen beiden durch die obigen Gleichungen. Eine Function wie $f_{n}$ oder $f_{n-1}$ ist aber eine lineare Verbindung von ganzen Potenzen der $\xi$ mit resp.

$$
\frac{(n+1)(n+2) \ldots(n+p-1)}{1.2 \ldots(p-1)}, \frac{n(n+1) \ldots(n+p-2)}{1.2 \ldots(p-1)}
$$

Constanten; hieraus folgt, dass $V$-wirklich nur $\sigma$ Constante, und zwar in der oben angegebenen Art enthalten kann. 
1.

$$
\begin{aligned}
\frac{1}{\left(1-2 r z+r^{2}\right)^{\frac{p-1}{2}}} & =\frac{\sqrt{ } \pi}{\Pi \frac{p-3}{2}} \sum_{n=0}^{n=\infty} r^{n} I^{n}[p, z] \quad(p>1) \\
-\log \left(1-2 r z+r^{2}\right) & =\sqrt{ } \pi \sum_{n=1}^{n=\infty} r^{n} I^{n}[1, z] \\
n I^{n}[1, z] & =\frac{1}{\sqrt{ } \pi} \text { für } n=0 \\
I^{n}[1, z] & =\frac{2}{n \sqrt{ } \pi} \cos (n \cdot \arccos z)=C^{n}(z) \quad(n>0) \\
I^{n}[2, z] & =P^{n}(z) \\
I^{n}[p, 1] & =\frac{\Pi^{\frac{p-3}{2}}}{\sqrt{ } \pi} \frac{\Pi(p+n-2)}{\Pi n \Pi(p-2)}
\end{aligned}
$$

II. $\mathfrak{J}_{\nu}^{n}[p, z]=z^{n-v}-\frac{(n-v)(n-\nu-1)}{2(2 n+p-3)} z^{n-\nu-2}+\frac{(n-v)(n-\nu-1)(n-\nu-2)(n-\nu-3)}{2.4(2 n+p-3)(2 n+p-5)} z^{n-v-4}-$ etc.

$I_{\nu}^{n}[p, z]=\left(\sqrt{z^{2}-1}\right)^{\nu} \mathfrak{\Im}_{\nu}^{n}[p, z]$

$I_{0}^{n}[p, z]=\mathfrak{S}_{i}^{n}[p, z] \quad I_{\nu}^{n}[2, z]=P_{\nu}^{n}(z)$

$\mathfrak{J}_{v}^{n}[p, z]=\mathfrak{\Im}_{\nu+m}^{n+m}[p-2 m, z]=\mathfrak{\Im}_{0}^{n-v}[p+2 v, z]=I_{0}^{n-v}[p+2 v, z]$

$I^{n}[p, z]=\frac{2^{n}}{\sqrt{ } \pi} \frac{\Pi \frac{2 n+p-3}{2}}{\Pi n} I_{0}^{n}[p, z]$

III.

$$
\begin{aligned}
\frac{d^{m} I^{n}[p, z]}{d z^{m}} & =2^{m} I^{n-m}[p+2 m, z] \\
2^{\varpi} I^{n}[2 \widetilde{\omega}+1, z] & =\frac{d^{\varpi} C^{n+\varpi}(z)}{d z^{\varpi}} \\
2^{\varpi} I^{n}[2 \widetilde{\omega}+2, z] & =\frac{d^{\varpi} P^{n+\varpi}(z)}{d z^{\varpi}} \\
2^{n-1} C^{n}(z) & =\frac{\sqrt{z^{2}-1}}{n \Pi \frac{2 n-1}{2}} \frac{d^{n}\left(\left(z^{2}-1\right)^{\frac{2 n-1}{2}}\right)}{d z^{n}} \\
2^{n} P^{n}(z) & =\frac{1}{\Pi n} \frac{d^{n}\left(\left(z^{2}-1\right)^{n}\right)}{d z^{n}}
\end{aligned}
$$


IV.

$$
\begin{aligned}
& 1.3 .5 \ldots(2 n-1)=\frac{2^{n}}{\sqrt{ } \pi} \Pi \frac{2 n-1}{2} \\
& \Pi \frac{n}{2} \Pi \frac{n-1}{2}=\frac{\Pi n}{2^{n}} \sqrt{ } \pi \\
& \int_{1}^{\pi} \sin ^{n} \varphi d \varphi=\frac{\Pi \frac{n-1}{2}}{\Pi \frac{n}{2}} \sqrt{ } \pi
\end{aligned}
$$

$n$ ganz und positiv.

V.

$$
\begin{gathered}
\frac{\left(z^{2}-1\right)^{\varpi}}{\Pi(n+\varpi)} \frac{d^{n+\varpi}\left(z^{2}-1\right)^{n}}{d z^{n+\varpi}}=\frac{1}{\Pi(n-\varpi)} \frac{d^{n-\varpi}\left(z^{2}-1\right)^{n}}{d z^{n-\varpi}} \\
\frac{\left(z^{2}-1\right)^{\frac{2 \varpi-1}{2}}}{n \Pi(n+\varpi-1)} \frac{d^{\varpi}}{d z^{\varpi}} \cdot\left[\sqrt{z^{2}-1} \frac{d^{n}\left(z^{2}-1\right)^{\frac{2 n-1}{2}}}{d z^{n}}\right]=\frac{d^{n-\varpi}\left(z^{2}-1\right)^{\frac{2 n-1}{2}}}{\Pi(n-\varpi) d z^{n-\varpi}}
\end{gathered}
$$

VI. $\quad I^{n}[p, \cos \gamma]=\frac{\Pi \frac{p-2}{2} \Pi(p+n-2)}{\pi \Pi n \Pi(p-2)} \int_{0}^{\pi}(\cos \gamma+i \sin \gamma \cos \theta)^{n} \sin ^{p-2} \theta d \theta$

$$
=\frac{\Pi \frac{p-2}{2} \Pi(p+n-2)}{\pi \Pi n \Pi(p-2)} \int_{0}^{\pi} \frac{\sin ^{p-2} \theta d \theta}{(\cos \gamma+i \sin \gamma \cos \theta)^{n+p-1}}
$$

$\int_{0}^{\pi}(\cos \gamma+i \sin \gamma \cos \theta)^{n} \sin ^{p} \theta d \theta=\frac{\Pi \frac{p-1}{2} \Pi \frac{2 n+p-1}{2}}{\Pi(p+n)} 2^{p+n} I_{0}^{n}[p+2, \cos \gamma]$

VII.

$$
\begin{gathered}
\int_{-1}^{1} I_{\nu}^{n}[p, z] I_{\nu}^{n}[p, z]\left(z^{2}-1\right)^{\frac{p-2}{2}} d z=0 \quad(m<n) \\
c^{n}[p]=\int_{-1}^{1}\left(I^{n}[p, z]\right)^{2}\left(z^{2}-1\right)^{\frac{p-2}{2}} d z \\
c_{\nu}^{n}[p]=\int_{-1}^{1}\left(I_{\nu}^{n}[p, z]\right)^{2}\left(z^{2}-1\right)^{\frac{p-2}{2}} d z \\
c^{n}[p]=\left(-\frac{1}{4}\right)^{\frac{p-2}{2}} \frac{2}{2 n+p-1} \frac{\Pi(n+p-2)}{\Pi n} \\
\frac{2^{2 n+p-1}}{\pi} c_{\nu}^{n}[p]=(-1)^{\frac{p+2 \nu-2}{2}}(2 n+p-1) \frac{\Pi(n-\nu) \Pi(n+\nu+p-2)}{\left(\Pi \frac{2 n+p-1}{2}\right)^{2}}
\end{gathered}
$$

Ausnahme: $n^{2} c^{n}[1]=-i$ für $n=0$. 


$$
\begin{aligned}
& \text { VIII. } \frac{\left(x+z \sqrt{x^{2}-1}\right)^{n}}{\Pi n \Pi \frac{2 n+p-2}{2}}=2^{p+n-2} \sum_{\nu=0}^{\nu=n} \frac{2 \nu+p-1}{\Pi(n-v) \Pi(n+\nu+p-1)} I_{\nu}^{n}[p+1, x] I^{\nu}[p, z] \\
& I^{n}[p, \cos \gamma]=\sum_{\nu=0}^{\nu=n} k_{\nu}^{n}[p] I_{\nu}^{n}[p, \cos \eta] I_{\nu}^{n}[p, \cos \psi] I^{\nu}\left[p-1, \cos \gamma_{1}\right] \\
& (-1)^{\nu} k_{\nu}^{n}[p]=\frac{(2 \nu+p-2)}{\sqrt{ } \pi} 2^{2 n+p-3} \frac{\left(\Pi \frac{2 n+p-3}{2}\right)^{2}}{\Pi(n-\nu) \Pi(n+\nu+p-2)} \\
& \cos \gamma=\cos \eta \cos \psi+\sin \eta \sin \psi \cos \gamma_{1} \\
& k_{\nu}^{n}[p] c_{\nu}^{n}[p]=(-1)^{\frac{p-2}{2}} \frac{2 \nu+p-2}{2 n+p-1} \sqrt{ }
\end{aligned}
$$$$
\frac{1}{\sqrt{ } \pi} \int_{0}^{\pi} I^{n}[p, \cos \gamma] \sin ^{p-2} \gamma_{1} d \gamma_{1}=2^{p-2} \frac{\Pi n \Pi \frac{p-3}{2}}{\Pi(n+p-2)} I^{n}[p, \cos \eta] I^{n}[p, \cos \psi]
$$$$
\int I^{m}\left[p, a x+a_{1} x_{1}+\text { etc. }+a_{p} x_{p}\right] I^{n}\left[p, b x+b_{1} x_{1}+\text { etc. }+b_{p} x_{p}\right] \frac{d x d x_{1} \ldots d x_{p-1}}{x_{p}}=0(m<n)
$$$$
=\frac{4 \pi^{\frac{p}{2}}}{2 n+p-1} I^{n}\left[p, a b+a_{1} b_{1}+\text { etc. }+a_{p} b_{p}\right] \quad(m=n)
$$$$
\text { wenn } a^{2}+a_{1}^{2}+\text { etc. }+a_{p}^{2}=b^{2}+b_{1}^{2}+\text { etc. }+b_{p}^{2}=1
$$$$
x^{2}+x_{1}^{2}+\cdots+x_{p}^{2}=1 \text {. }
$$

Halle, November 1862. 